\title{
Programmed Switching of Single Polymer Conformation on DNA Origami
}

Abhichart Krissanaprasit, Mikael Madsen, Jakob Bach Knudsen, Daniel Gudnason, Werasak

Surareungchai, Victoria Birkedal and Kurt Vesterager Gothelf*

\section{Table of contents}

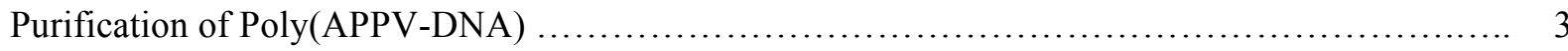

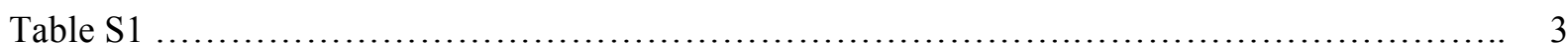

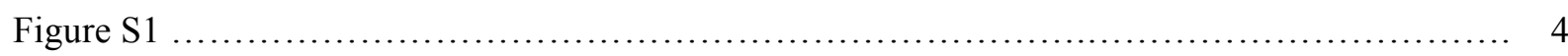

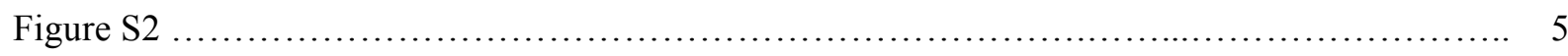

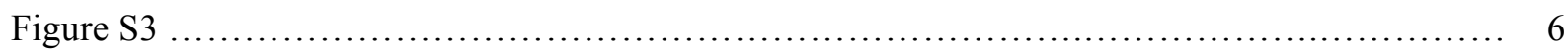

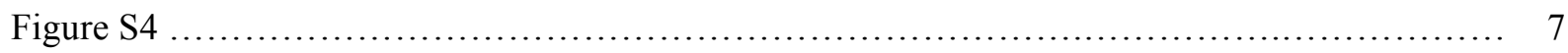

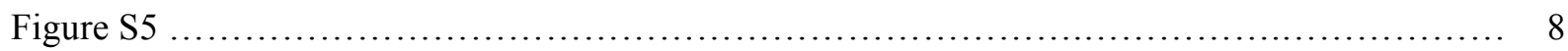

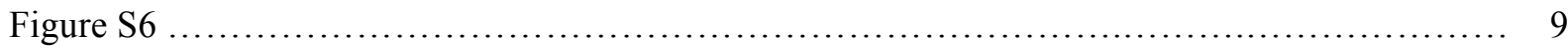

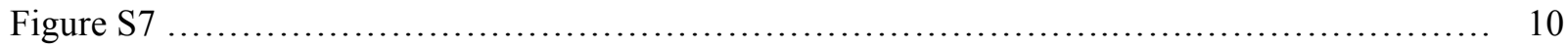

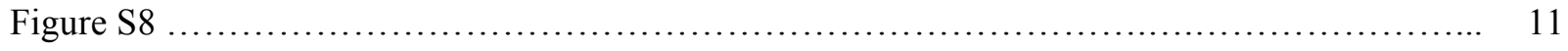

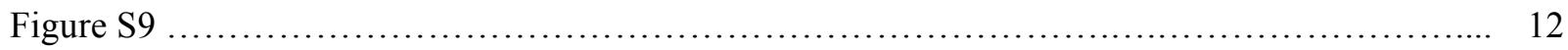

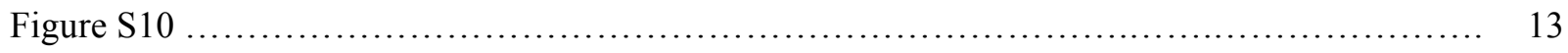

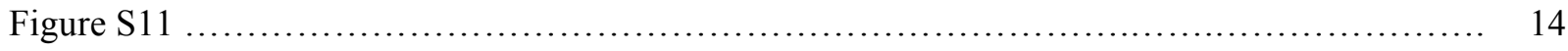

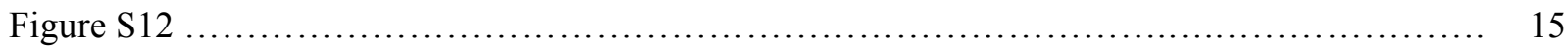

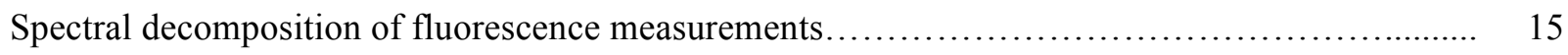

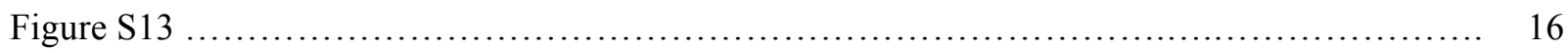

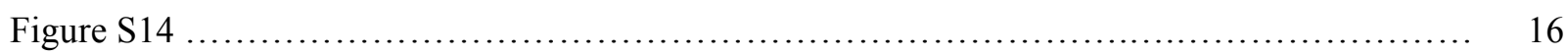

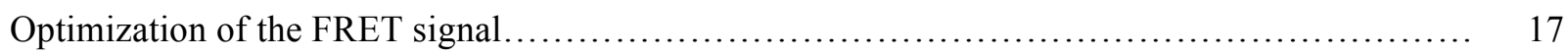

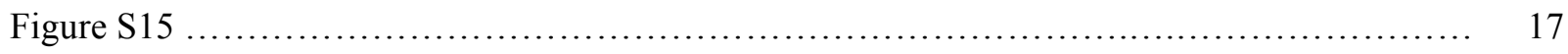

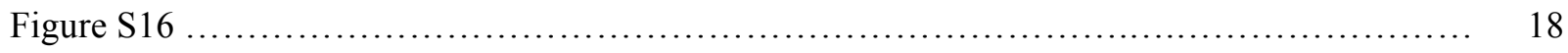

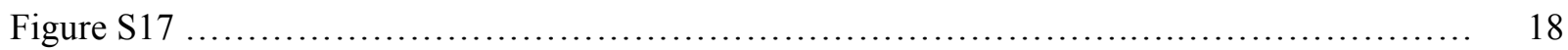




\section{Supporting Information}

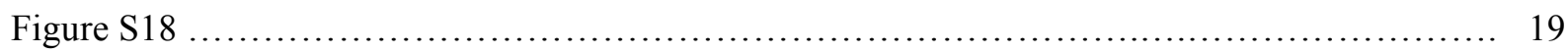

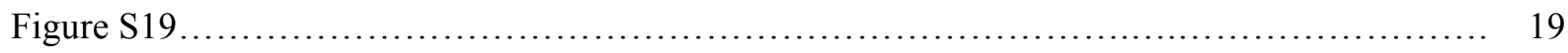

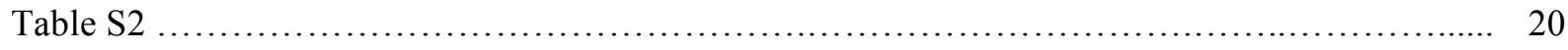

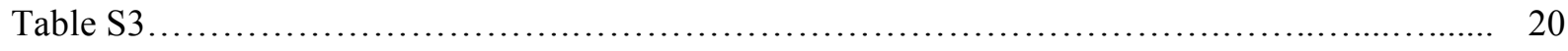

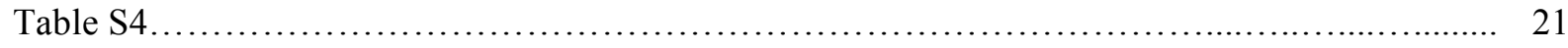

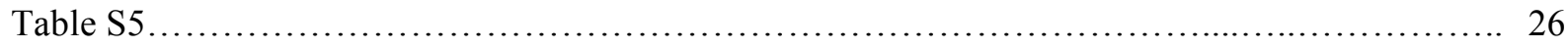

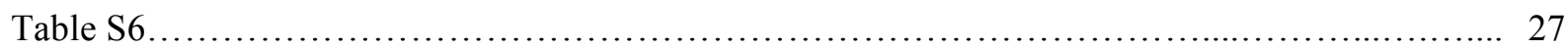

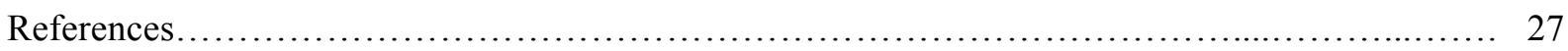




\section{Purification of Poly(APPV-DNA)}

Poly(APPV-DNA) was purified using size exclusion chromatography according to the procedure described in Knudsen et al. ${ }^{1}$

Typically, the CPG from one synthesis column was transferred to a polypropylene tube with a screw cap. Then $0.6 \mathrm{~mL}$ of AMA (1:1 aqueous methylamine $40 \%$ /ammonium hydroxide $30-33 \%)$ was added, and the polymer-DNA conjugates were cleaved from the solid support by incubation at $65^{\circ} \mathrm{C}$ for 15 minutes. The AMA polymer solution was cooled at $-20{ }^{\circ} \mathrm{C}$ for 5 minutes before transferring to the column. The size exclusion column was manually packed by transferring $13 \mathrm{~mL}$ of sepharose CL-2B dispersion (Sigma Aldrich) to an empty PD-10 column (GE-Healthcare) with a frit in the bottom. The column was left until it stopped dripping, and at this point a frit was carefully added to the top of the column. An amount of $20 \mathrm{~mL}$ of $0.05 \mathrm{M}$ TEAA was eluted through the column in portions of $4 \mathrm{~mL}$. If smaller volumes were used, sepharose was observed in the isolated polymer-DNA conjugates. The AMA polymer solution was then directly added to the column. When the solution had migrated into the column, poly(APPV-DNA) was eluted in $1 \mathrm{~mL}$ fractions using $0.05 \mathrm{M}$ TEAA. Typically the polymers start eluting in fraction 3 or 4 , and elute through to fraction 8 . The isolated fractions were lyophilized, redissolved in MilliQ water and lyophilized again to afford a fluffy pink solid. In fraction 7 and 8 single-stranded DNA is observed along with the polymer, and these fractions were never used in polymer experiments. In the experiments described herein, only fractions 4 and 5 were used.

The obtained fractions of poly(APPV-DNA) were analyzed using GPC as described in Kundsen et al. ${ }^{1}$ The molecular weights were determined using a $\mathrm{dn} / \mathrm{dc}$ value of $0.1850 \mathrm{~mL} / \mathrm{g}$.

Table S1 Molecular weights and polydispersity index value (PDI) of poly(APPV-DNA) corresponding to the fractions collected from size exclusion chromatography

\begin{tabular}{|l|l|l|l|}
\hline Fraction & $M_{\mathrm{N}}(\mathrm{kDa})$ & $\mathrm{M}_{\mathrm{w}}(\mathrm{kDa})$ & PDI \\
\hline 3 & $2908.0( \pm 0.4 \%)$ & $3344.5( \pm 0.6 \%)$ & $1.150( \pm 0.7 \%)$ \\
\hline 4 & $2663.1( \pm 0.5 \%)$ & $3240.1( \pm 0.9 \%)$ & $1.217( \pm 1.0 \%)$ \\
\hline 5 & $1540.8( \pm 0.6 \%)$ & $1906.2( \pm 0.6 \%)$ & $1.237( \pm 0.8 \%)$ \\
\hline 6 & $1046.1( \pm 0.7 \%)$ & $1291.2( \pm 0.6 \%)$ & $1.234( \pm 1.0 \%)$ \\
\hline 7 & $347.6( \pm 4.6 \%)$ & $724.8( \pm 1.0 \%)$ & $2.085( \pm 4.7 \%)$ \\
\hline 8 & $54.9( \pm 6.8 \%)$ & $343.9( \pm 1.1 \%)$ & $6.260( \pm 6.9 \%)$ \\
\hline
\end{tabular}

More details about the GPC characterization can be found in Kundsen J. B. et al. ${ }^{1}$ It should be noted that the difference between $\mathrm{M}_{\mathrm{W}}$ and $\mathrm{M}_{\mathrm{N}}$ and hence also the PDIs are underestimated due to the high molecular weights of poly(APPV-DNA) making it elute after one void volume of the GPC column. 
(A)

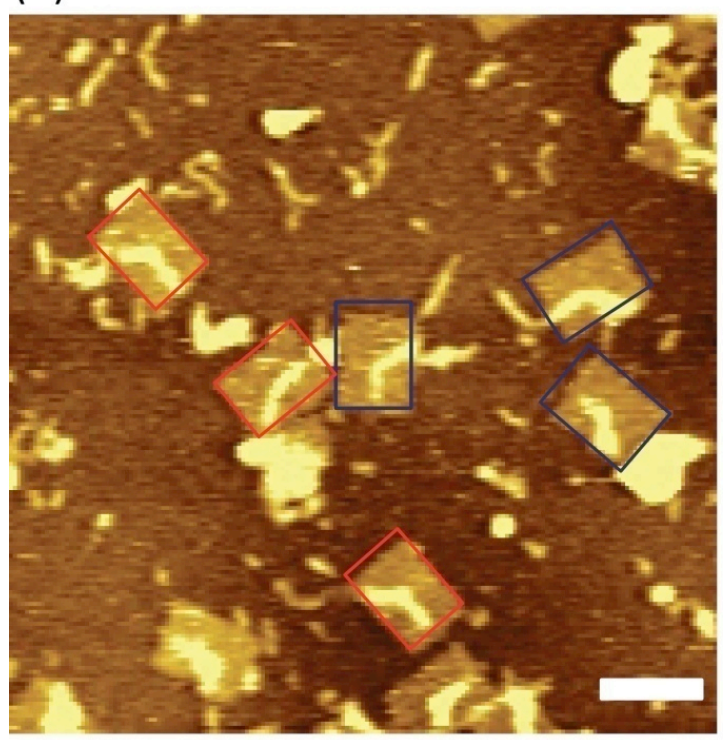

(C)

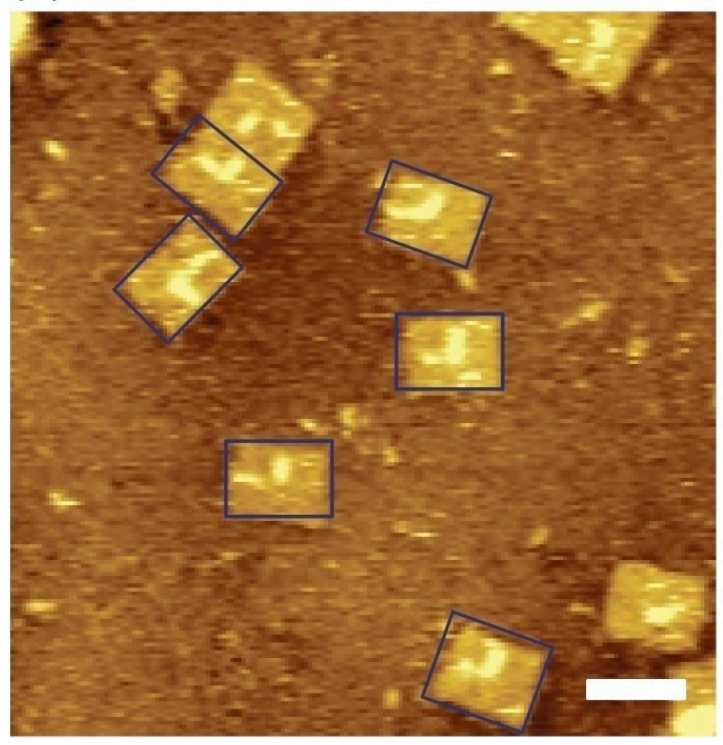

(B)

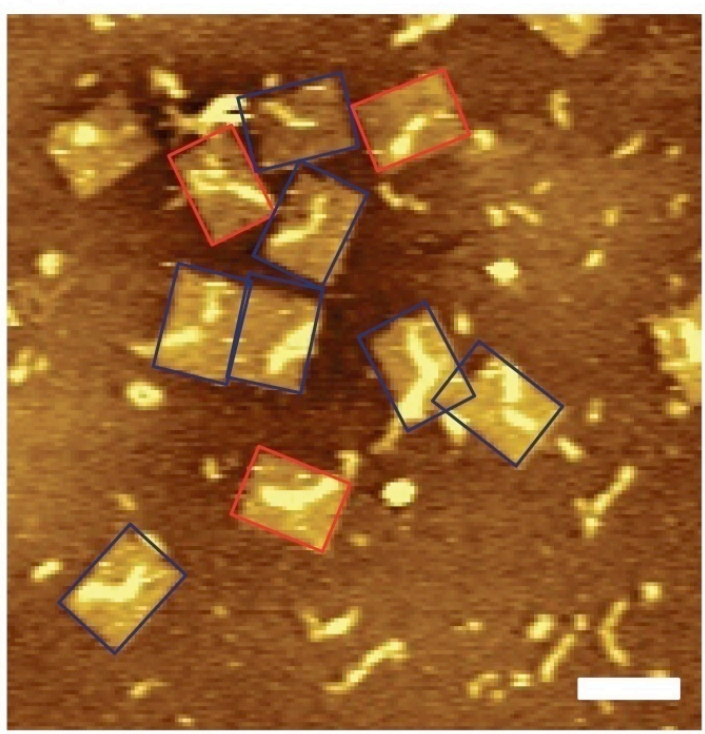

(D)

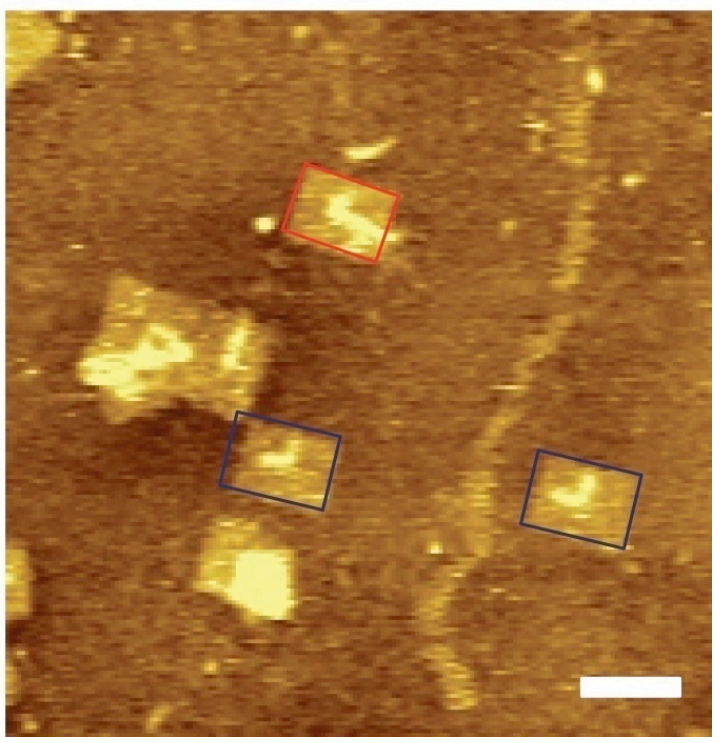

Figure S1 AFM images of poly(APPV-DNA) assembled on designed paths on DNA origami. (A-B) Rturned and (C-D) L-turned patterns. Dark blue and red rectangles represent DNA origami facing up and down on the mica surface, respectively. For statistical analysis, both DNA origami facing up and down have been counted. The majority of DNA origami on mica surface are facing up. Scale bar is $100 \mathrm{~nm}$. 


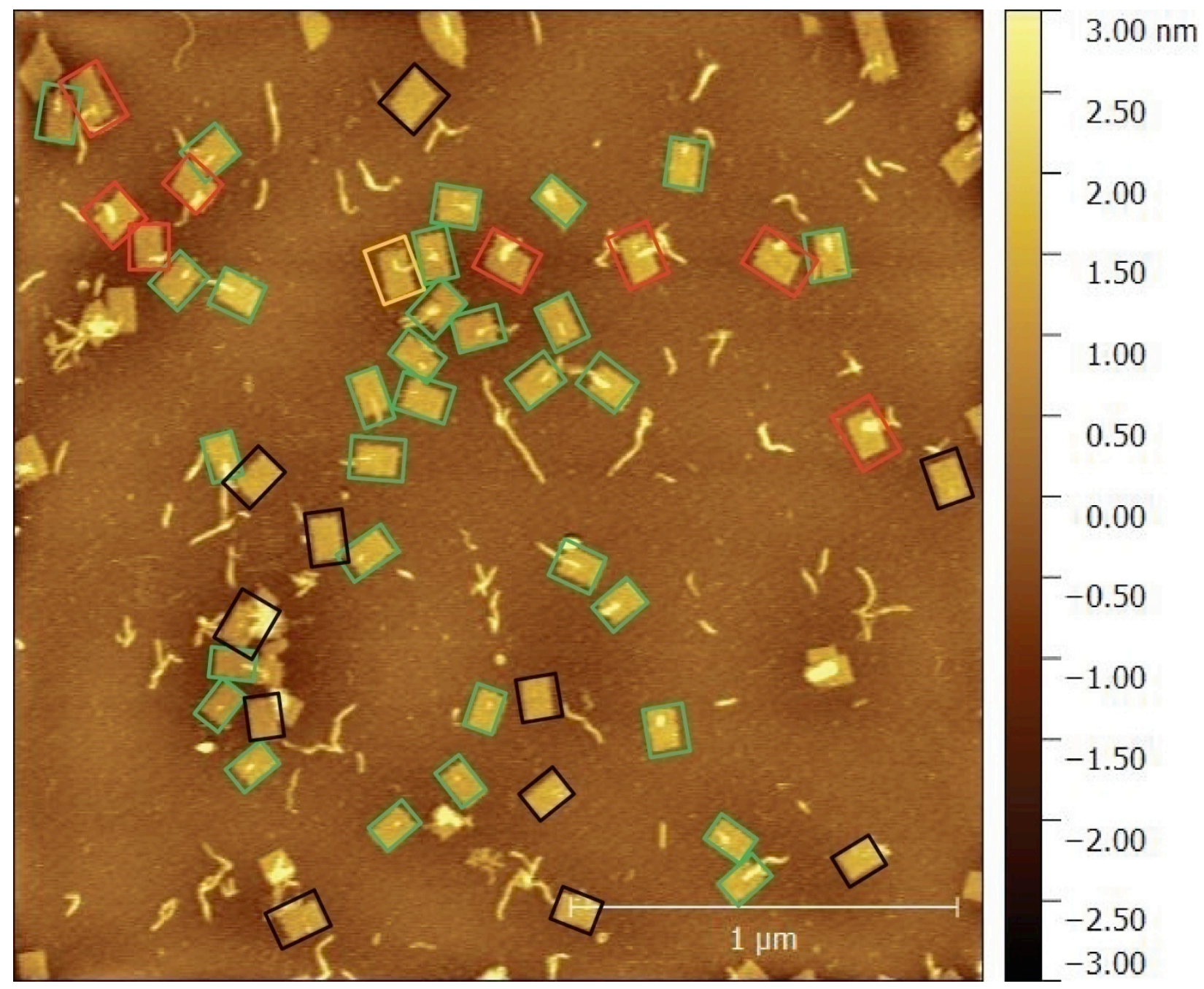

Figure S2 Topography AFM image of poly(APPV-DNA) alignment in a straight line pattern on DNA origami (green box). Plain origami, L-turned pattern and origami with misaligned polymer are represented in black, yellow and red boxes, respectively. 


\section{Supporting Information}

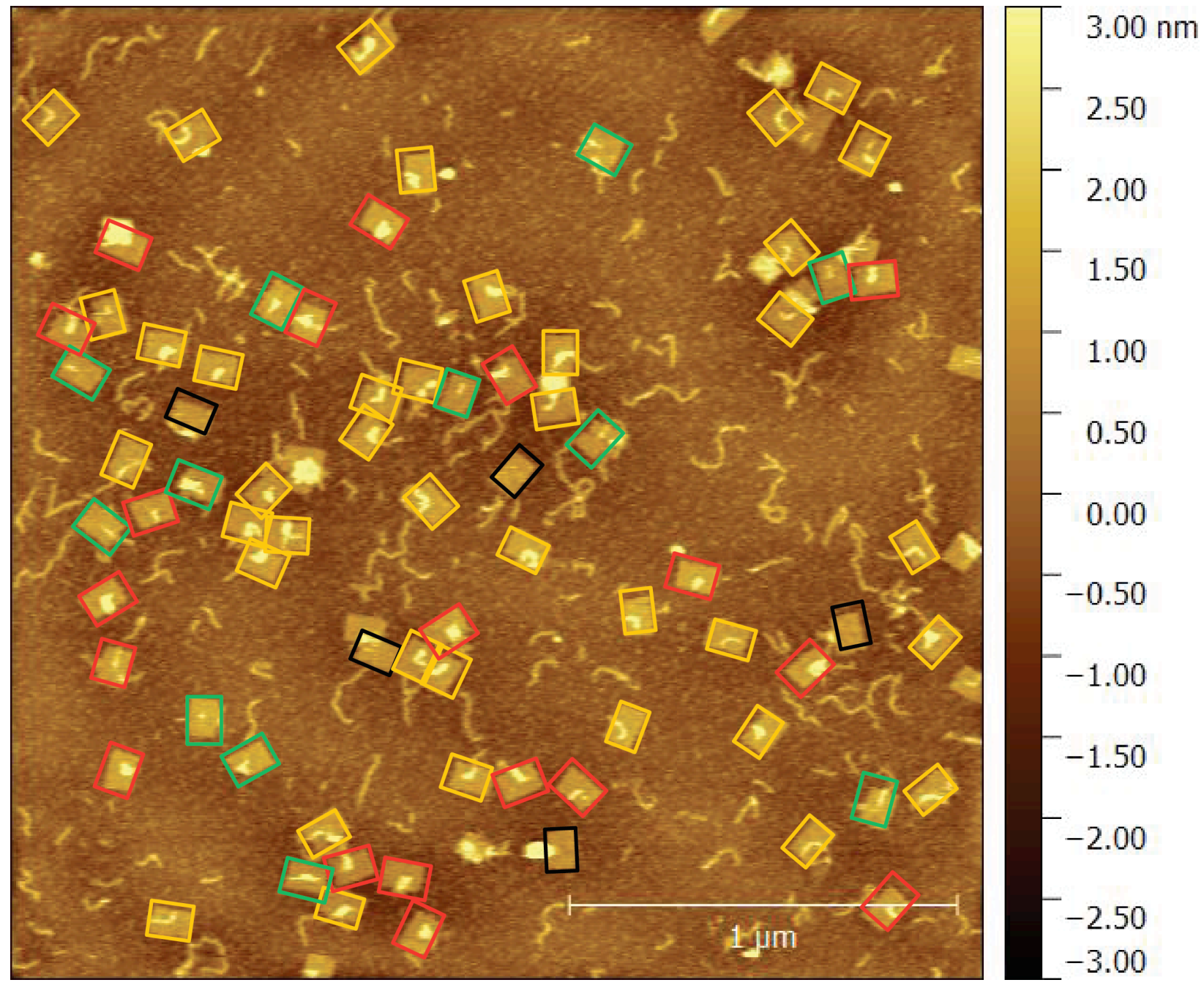

Figure S3 Topography AFM image of DNA origami containing the polymer in the L-turned pattern (yellow box). Black, green and red boxes represent plain origami, straight line pattern of polymer on DNA origami and origami with misaligned polymer, respectively. 


\section{Supporting Information}

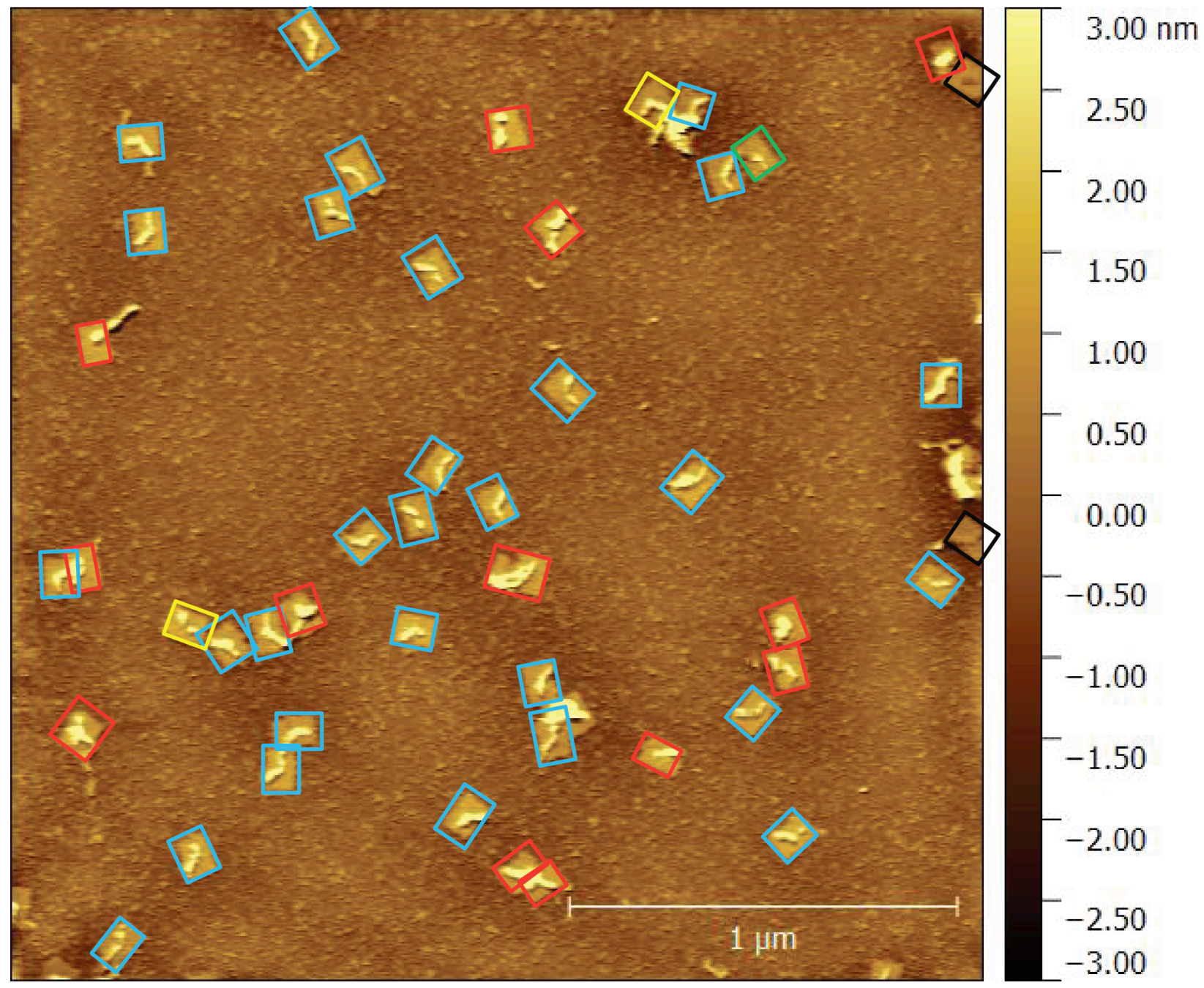

Figure S4 Topography AFM image of the R-turned pattern (blue box). Polymer alignment in straight line, L-turned pattern, plain origami and origami with misaligned polymers are represented in green, yellow, black and red box, respectively. 


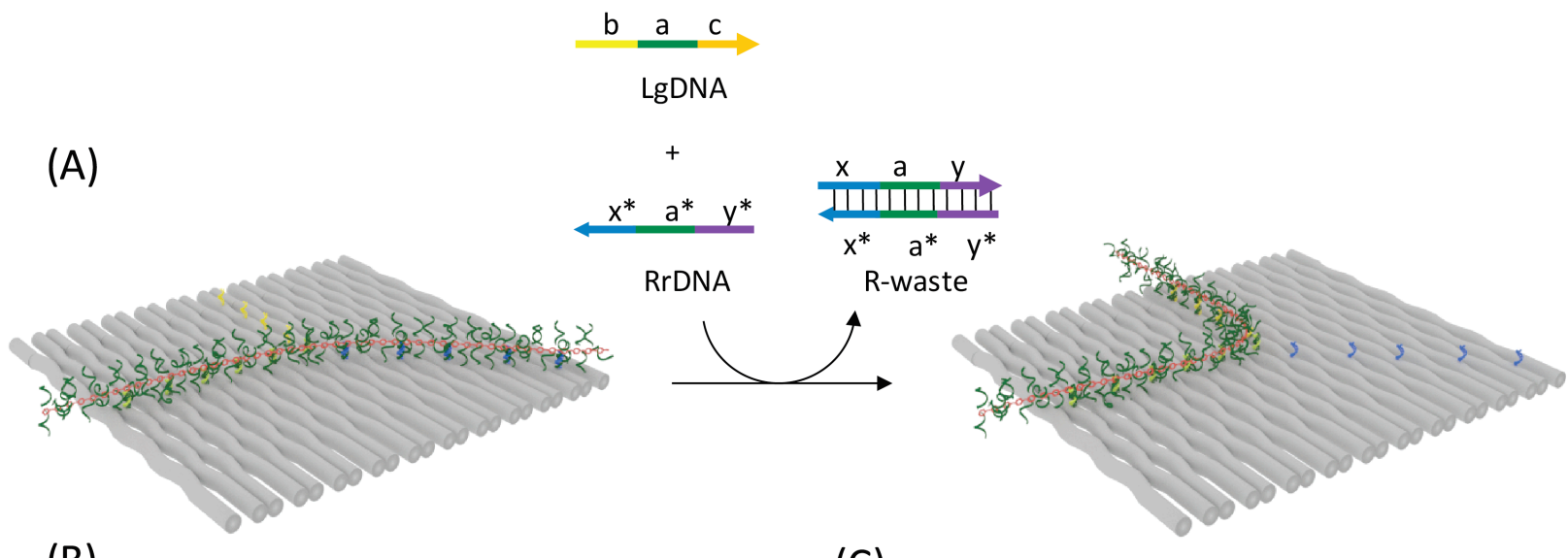

(B)

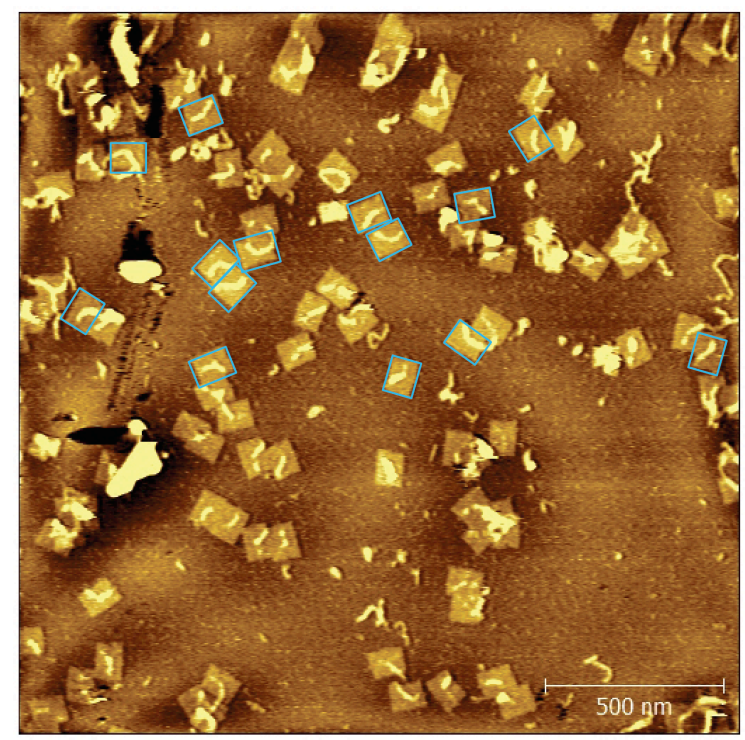

(C)

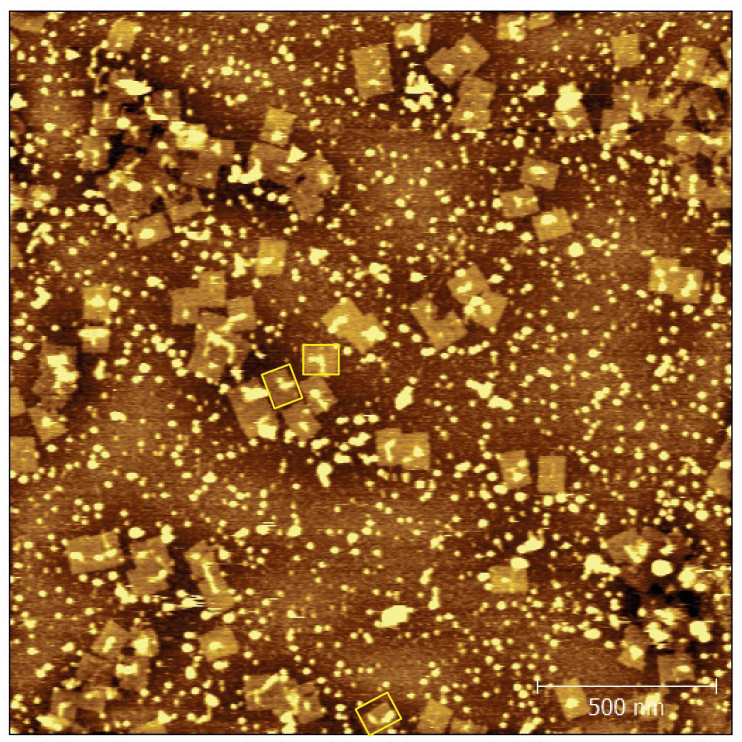

Figure S5 Programmed switching of poly(APPV-DNA) on a mica surface. (A) Schematic illustration of the conformation switching of the poly(APPV-DNA) from the R-turned to L-turned pattern. (B) Topography AFM image of poly(APPV-DNA) alignment in the R-turned conformation highlighted in blue boxes. (C) AFM image the polymer on DNA origami after switching from the R-turned to L-turned pattern. Poly(APPV-DNA) alignment in the L-turned pattern is shown in a yellow box. The yield of polymer switching on DNA origami on mica surface is quite low. A high number of small dots, probably salt crystals, were found after the switching process on mica surface; therefore, observation of further switching processes on mica was impossible. 


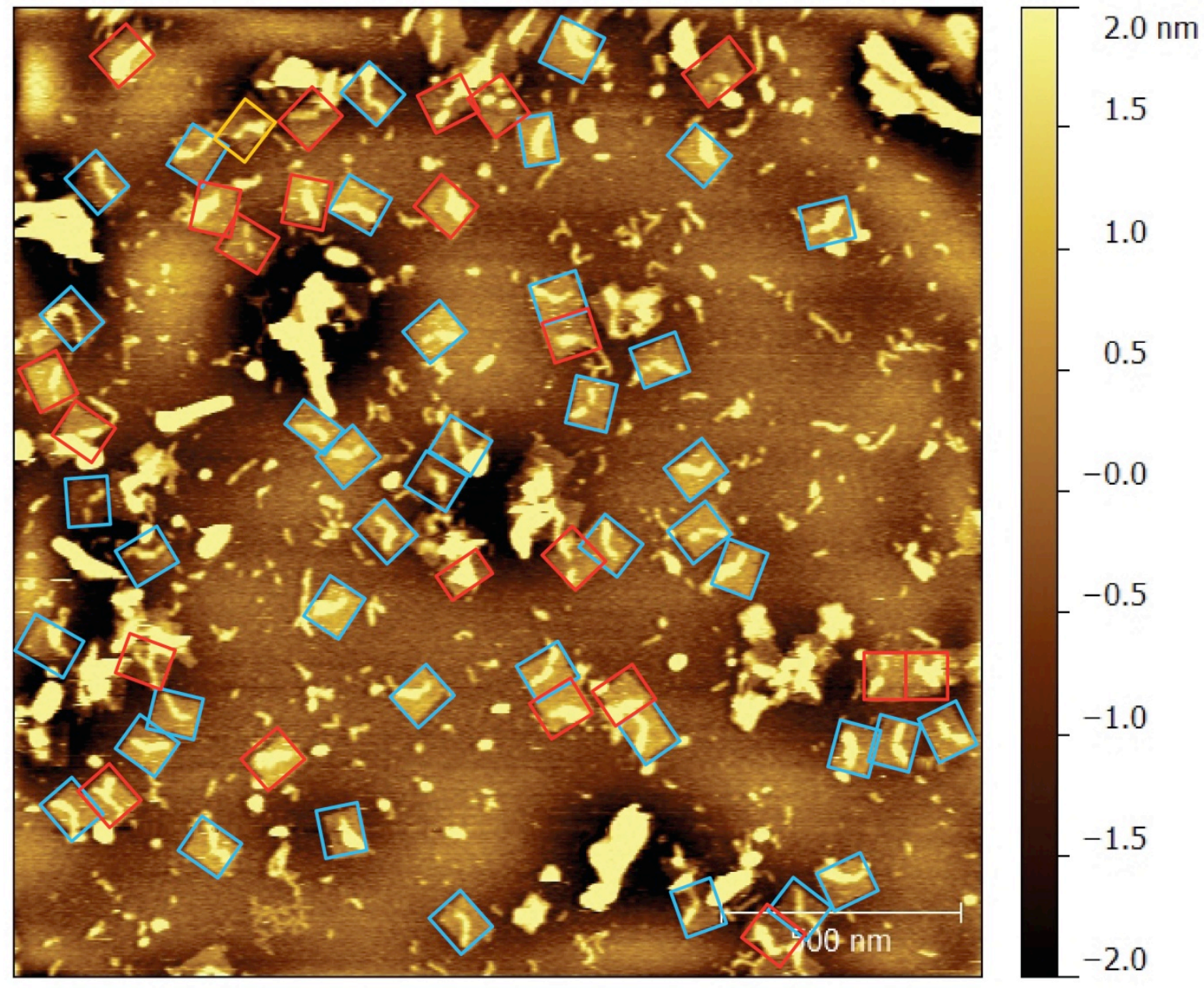

Figure S6 Programmed switching of poly(APPV-DNA) in solution. In the initial state of switching, the polymer is aligned in the R-turned pattern (blue box). The L-turned pattern and misaligned polymer patterns are represented in yellow and red box, respectively 


\section{Supporting Information}

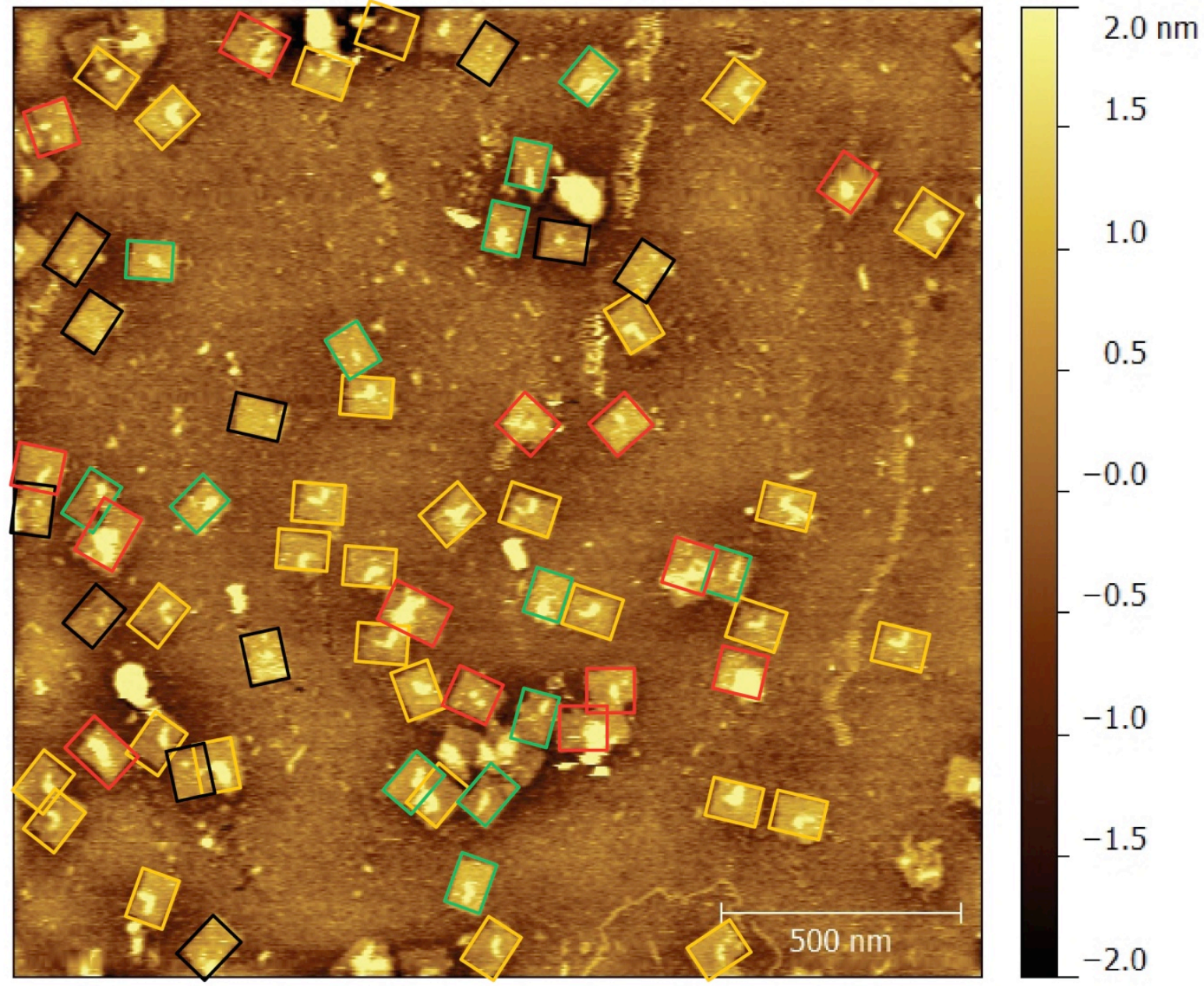

Figure S7 Topography AFM image of programmed switching of poly(APPV-DNA) in solution. After the first switching, the polymer is mainly aligned in the L-turned pattern (yellow box). Straight line, plain origami and misaligned polymer patterns are highlighted in green, black and red box, respectively. Most of free polymer was removed by spin-filter purification. 


\section{Supporting Information}

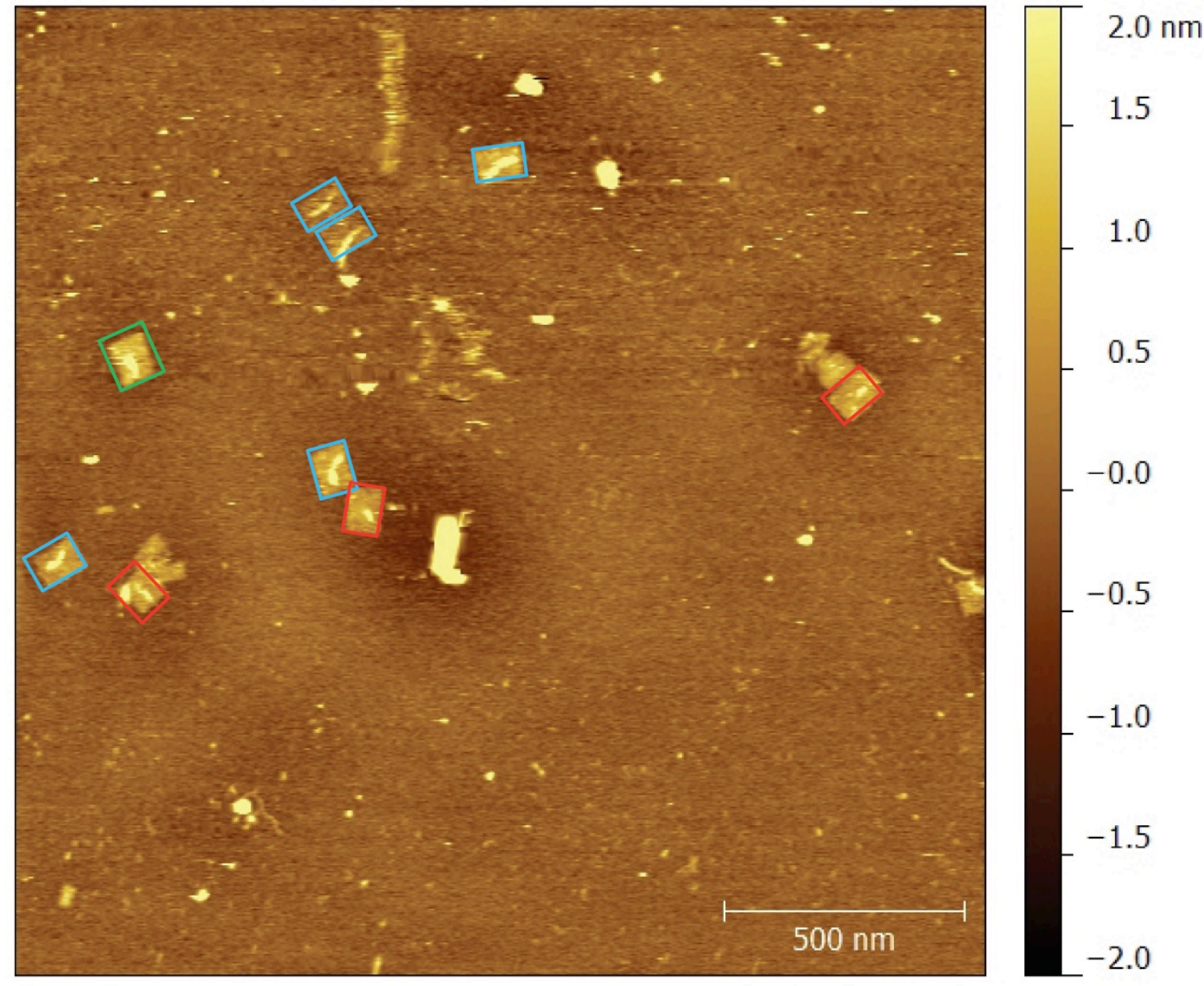

Figure S8 Topography AFM image of programmed switching of poly(APPV-DNA). After the $2^{\text {nd }}$ switching, the polymer is switched from the L-turned to the R-turned pattern (blue box). Straight line and misaligned polymer patterns are highlighted in green and red box, respectively. 


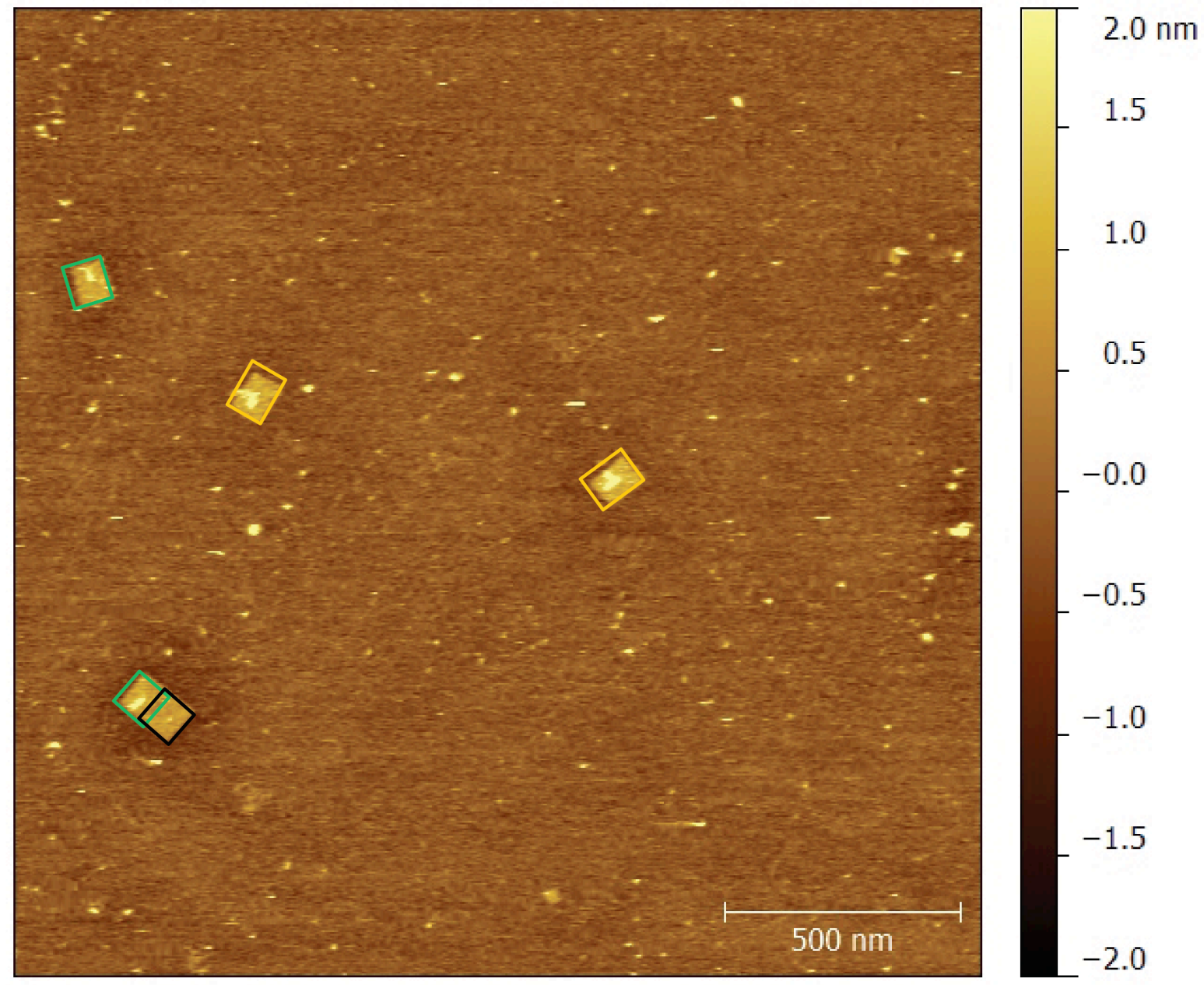

Figure S9 Topography AFM image of programmed switching of poly(APPV-DNA). After $3^{\text {rd }}$ switching the polymer is switched from the R-turned to the L-turned pattern (yellow box). Straight line patterns and plain origami are represented in green and black box, respectively. 

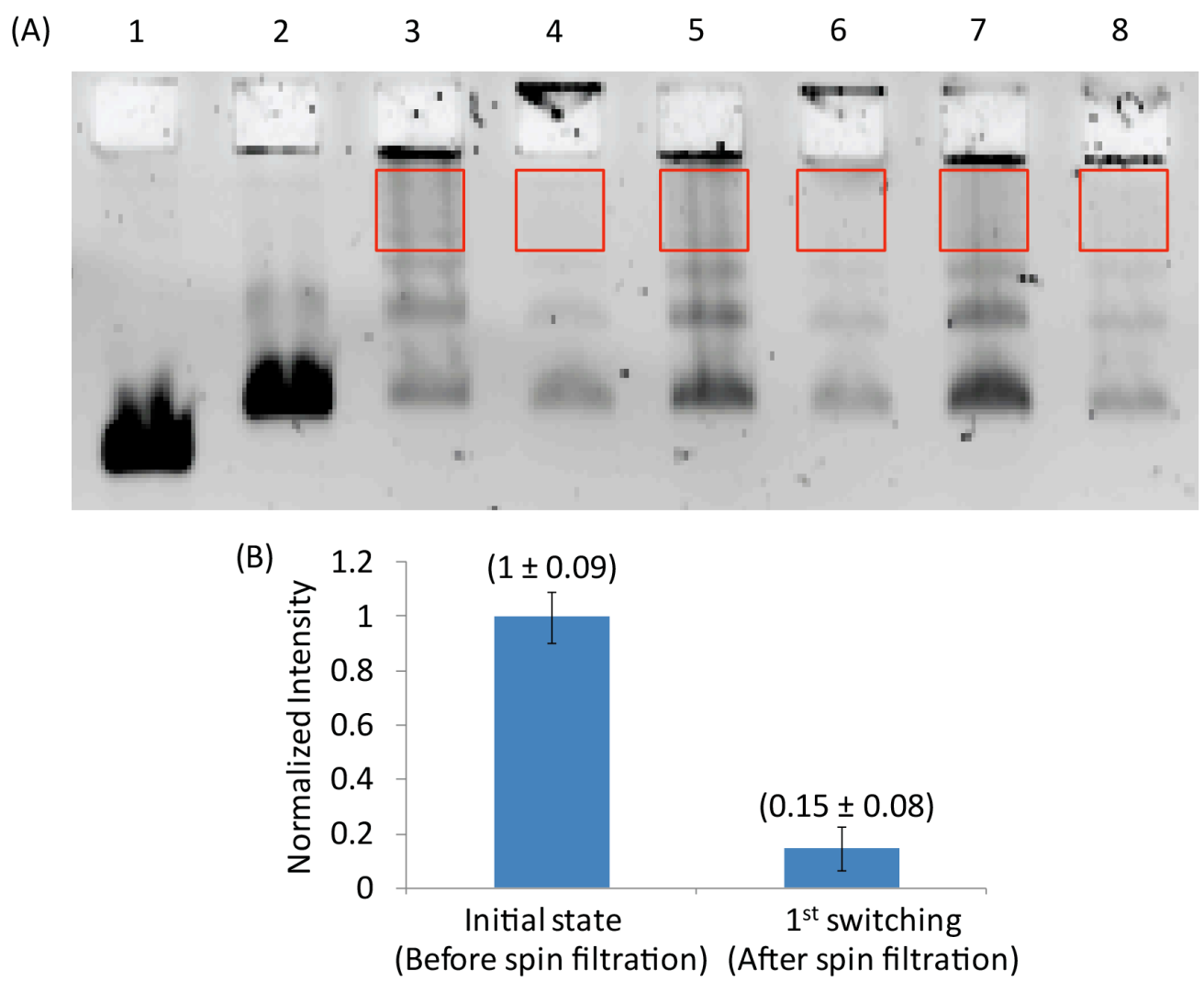

Figure S10 Estimation of the amount of poly(APPV-DNA) remaining after purification by spin filtration in a switching process. (A) Agarose gel electrophoresis of poly(APPV-DNA) aligned on DNA origami before and after spin filtration. Lane 1: M13mp18 scaffold. Lane 2: Assembled DNA origami. Lane 3, 5 and 7: Poly(APPV-DNA) aligned in the R-turned pattern on DNA origami (initial state) before spin filtration. Lane 4, 6 and 8: the $1^{\text {st }}$ switching, poly(APPV-DNA) in L-turned pattern after switching and spin filtration. In consistency with previous gel analysis data reported by Knudsen et al. most of the free polymers don't migrate from the well due to their large sizes. ${ }^{1}$ For quantification, we have only included the fraction of polymers that migrate from the wells since inclusion of the structures remaining in the wells provided unreliable results. The areas used for quantification of poly(APPV-DNA) are denoted with red boxes. It is, however, noted that the polymer bands in the bottom of the wells before spin filtration, are not observed after spin filtration. This further confirms that the polymers are removed during spin filtration. (B) Statistical analysis of remaining poly(APPV-DNA) before and after spin filtration. The values on the top of the bar are average and S.D. of normalized intensity, respectively. 


\section{Supporting Information}

(A)

(B)

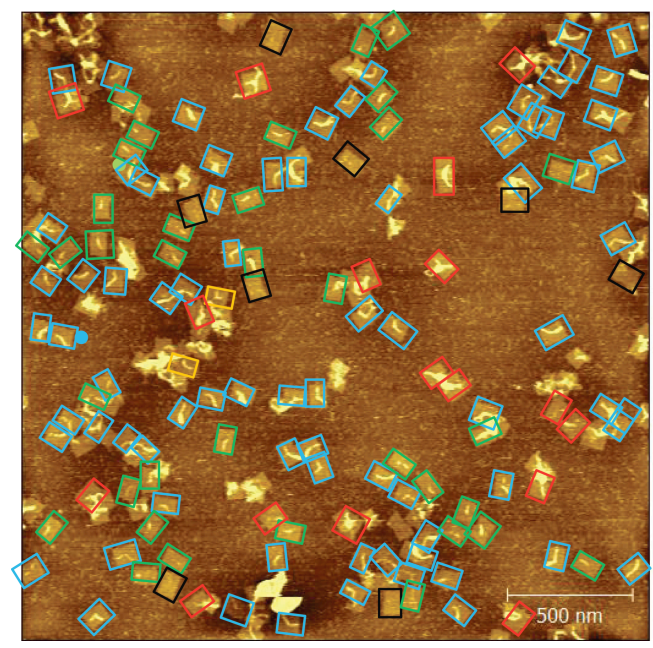

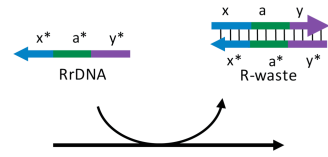

(C)
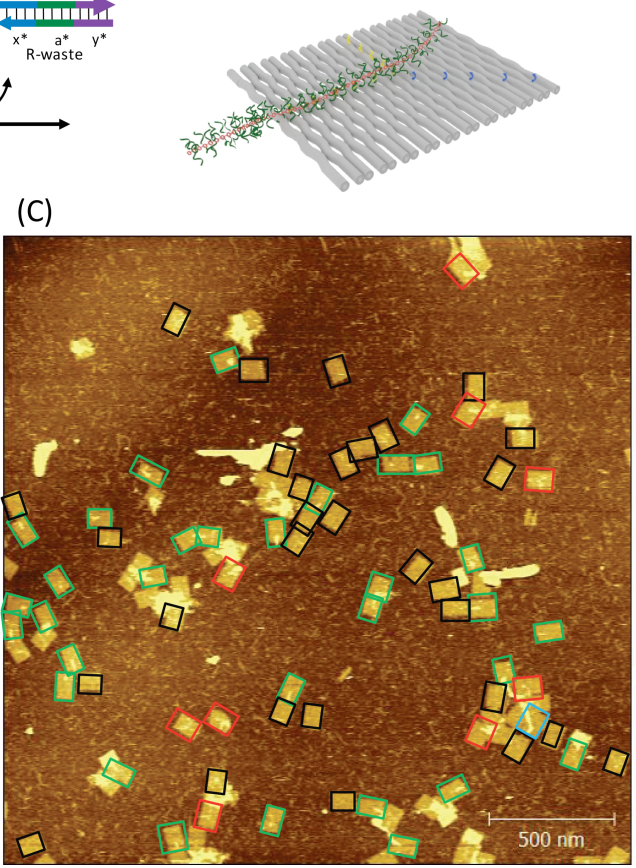

Figure S11 Topography AFM images of poly(APPV-DNA) linked on DNA origami track during switching conformation process. (A) Illustration of poly(APPV-DNA) permanently linked on DNA origami during switching conformation (B) In an initial state, poly(APPV-DNA) was aligned in R-turned pattern. (C) After adding RrDNA, the poly(APPV-DNA) was aligned in a straight line pattern on a linear hinge. Plain DNA origami represented in black boxes. Straight line, R-turned and L turned pattern are shown in green, blue and yellow boxes. Misaligned polymer patterns are highlighted in red box. Scale bar is $500 \mathrm{~nm}$. 


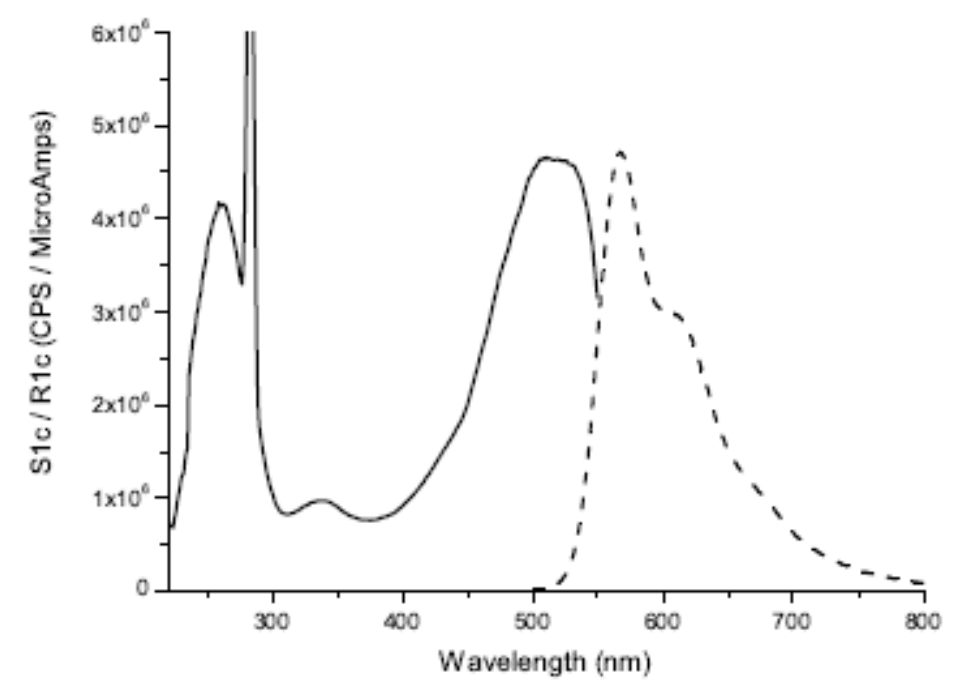

Figure S12 Excitation and emission spectra of poly(APPV-DNA) are represented in solid and dash line, respectively. The emission spectrum was recorded with a fixed excitation wavelength of $475 \mathrm{~nm}$, and the excitation spectrum was recorded with a fixed emission wavelength of $567 \mathrm{~nm}$. The high-intensity narrow peak observed at $283.5 \mathrm{~nm}$ in the excitation spectrum arises from half the fixed emission wavelength.

\section{Spectral decomposition of fluorescence measurements}

The fluorescence signal was decomposed with a reference spectra of poly(APPV-DNA) and acceptor 1 and 2 using the freeware program "a|e". ${ }^{2}$ Reference fluorescence spectra for poly(APPV-DNA) were obtained using DNA origami samples without additional dyes and the polymer was added to the solution and its conformation switched following the same procedure as for the data where the acceptor 1 and 2 were presented. Fluorescent spectra were recorded for each switch. In this manner changes in the spectral shape of the polymer contribution due to switching could be taken into account. The reference spectra for the dyes on the origami were obtained by measurements on origami samples with dyes attached in the absence of polymer.

Examples of spectral decomposition and fit quality for both L- and R-tuned patterns are presented in Figure S13-14. Visually it can be seen that the fits are good and the spectra can be well fitted by including contributions from poly(APPV-DNA) and acceptor 1 and acceptor 2 . This is further confirmed by the high $\mathrm{R}^{2}$ values of $\mathrm{R}^{2}=0.99983$ and $\mathrm{R}^{2}=0.99978$ for the $\mathrm{R}$ - and L-tuned patterns respectively, indicating little overall difference between fit and spectra in both cases. 


\section{Supporting Information}

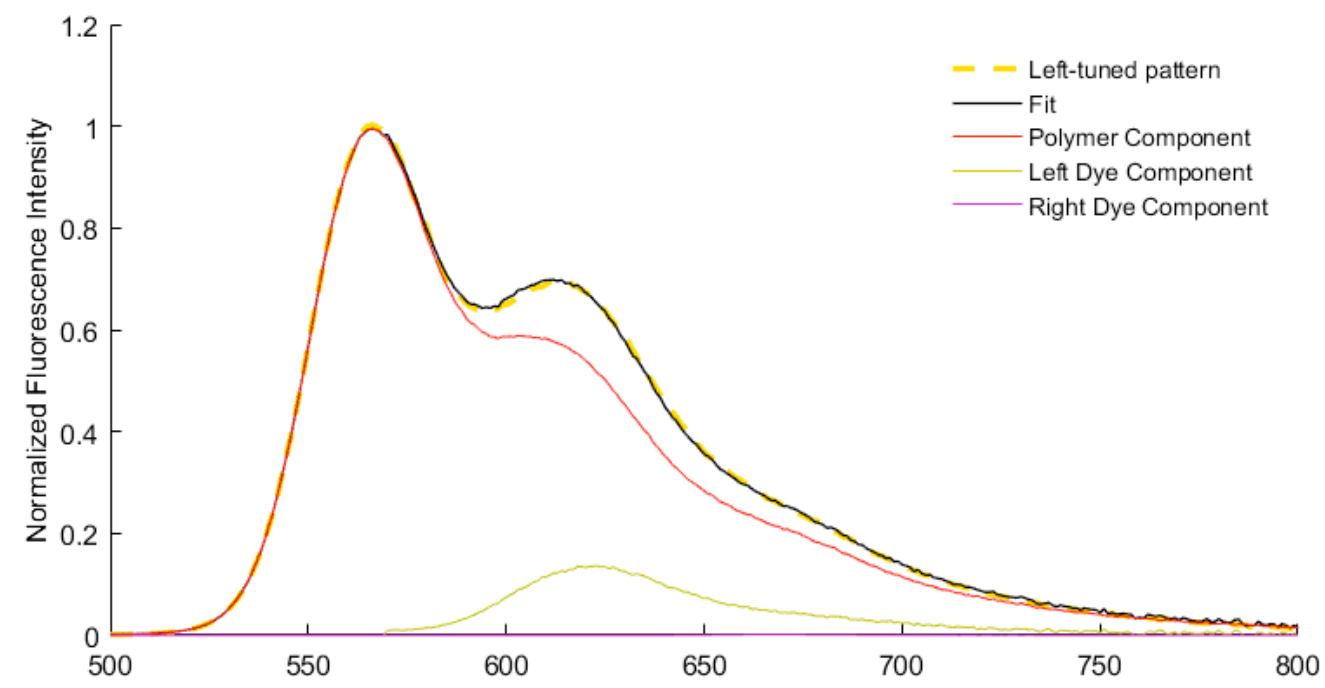

Figure S13 Example of emission spectra of the L-tuned pattern (dashed line) and references showing the measured data, the spectral decomposition components and the resulting fit. The coefficient of determination for the fit is $\mathrm{R}^{2}=0.99978$

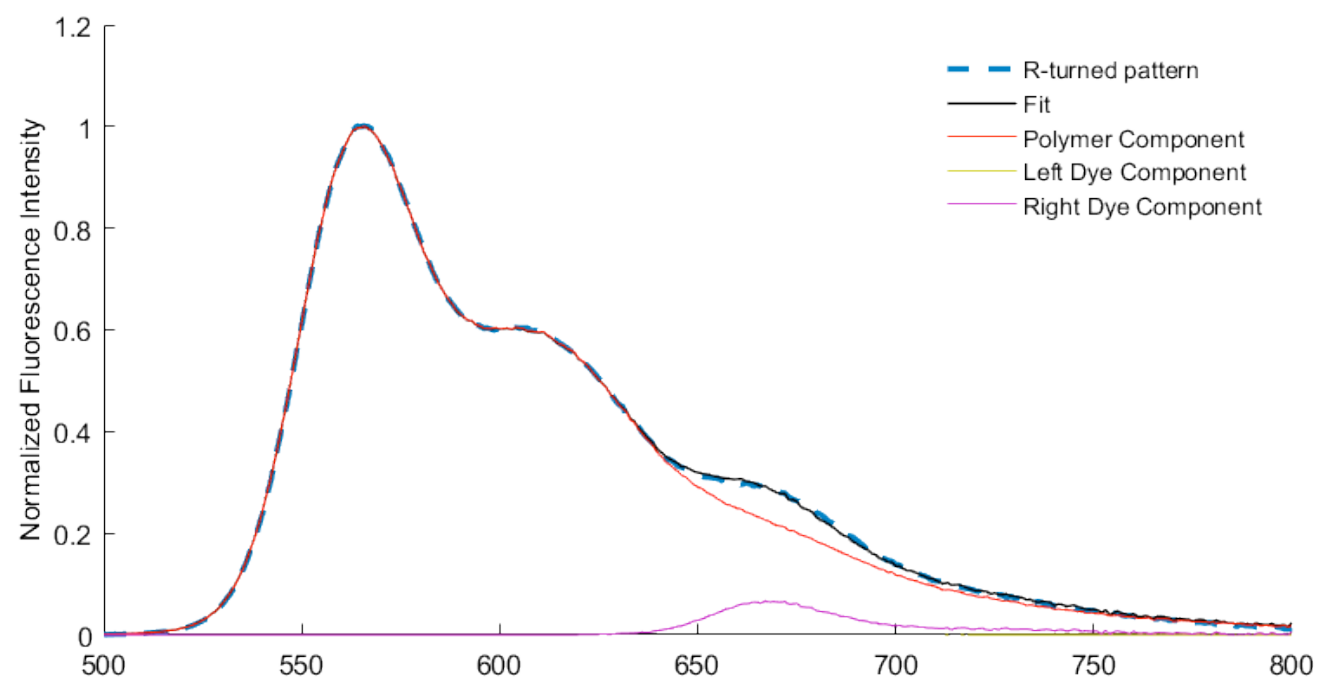

Figure S14 Example of emission spectra of the R-tuned pattern (dashed line) and references showing the measured data, the spectral decomposition components and the resulting fit. The coefficient of determination for the fit is $\mathrm{R}^{2}=0.99983$ 


\section{Supporting Information}

\section{Optimization of the FRET signal}

In the initial design for investigating the polymer conformation switching by FRET, acceptors 1 and 2 were placed at the end of the left track ( $30 \mathrm{~nm}$ away from middle track, calculated from design) and the right track (40 nm from middle track, calculated from design) respectively as shown in Figure S18. As presented in Figure S15, we performed six successive switching processes of the poly(APPV-DNA) conformation. However, the fluorescence intensities of acceptor 2 in the L-turned and R-turned conformations were not well-separated (Figure S15). Therefore, to increase the switching readout, optimization of acceptor 2 intensity was required. The fluorescence intensity of acceptor 2 is lower than that for acceptor 1 after FRET from poly(APPV-DNA), which is expected, since the spectral overlap between polymer emission and acceptor excitation is smaller for acceptor 2 than for acceptor 1 (Figure S16). Moreover, the linear distance from the middle track to acceptor 2 is larger than that to acceptor 1. In order to increase the fluorescence intensity of acceptor 2, we studied the distance-dependent fluorescence intensity by placing an acceptor 2-modified staple strand at different distances from the middle track (30, 35 and $40 \mathrm{~nm}$ ). Positioning acceptor 2 at the shortest distance of $30 \mathrm{~nm}$ provided the highest fluorescence intensity as shown in Figure S17. The optimized set-up provides better fluorescence signal for acceptor 2. The further experiments with nanomechanical switching of poly(APPV-DNA) on DNA origami and timedependent FRET were carried out with the optimized set-up.

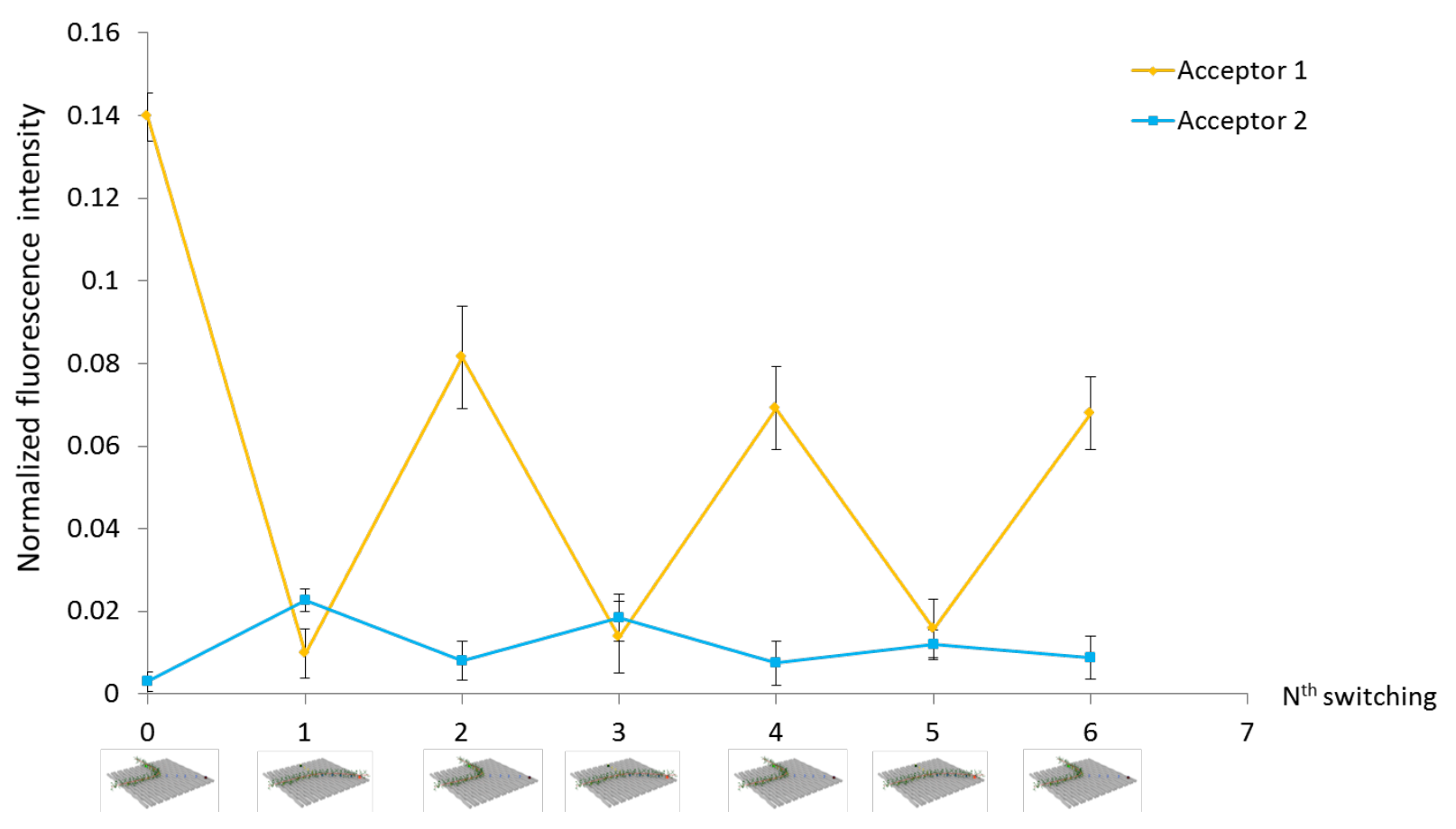


Figure S15 FRET study of switching on DNA origami containing acceptor 1 and acceptor 2, when placed $30 \mathrm{~nm}$ and $40 \mathrm{~nm}$ away from middle track respectively.

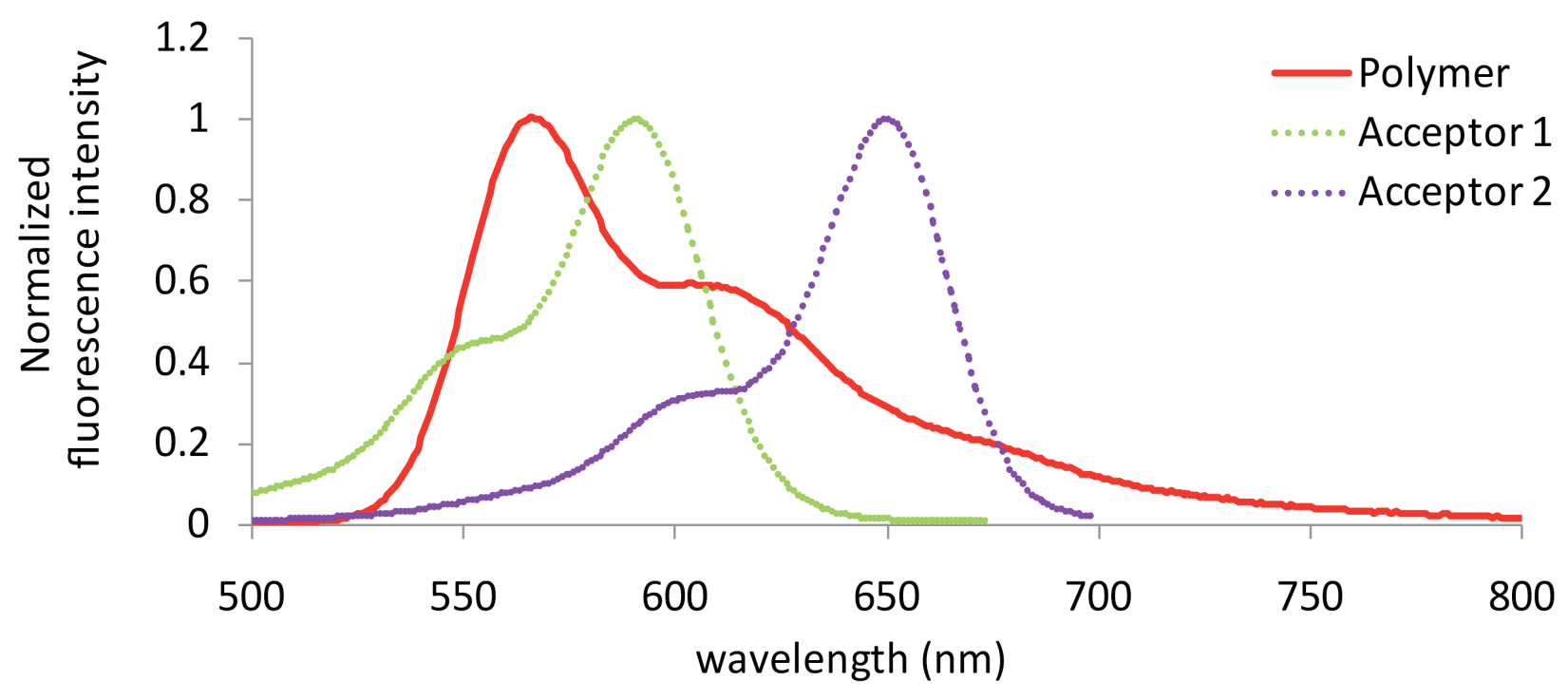

Figure S16 Fluorescence spectra of poly(APPV-DNA) and acceptor. Red solid line represents emission spectrum of poly(APPV-DNA) where was excited at $475 \mathrm{~nm}$. Green and purple dot line represent excitation spectra of acceptor 1 and 2, respectively.

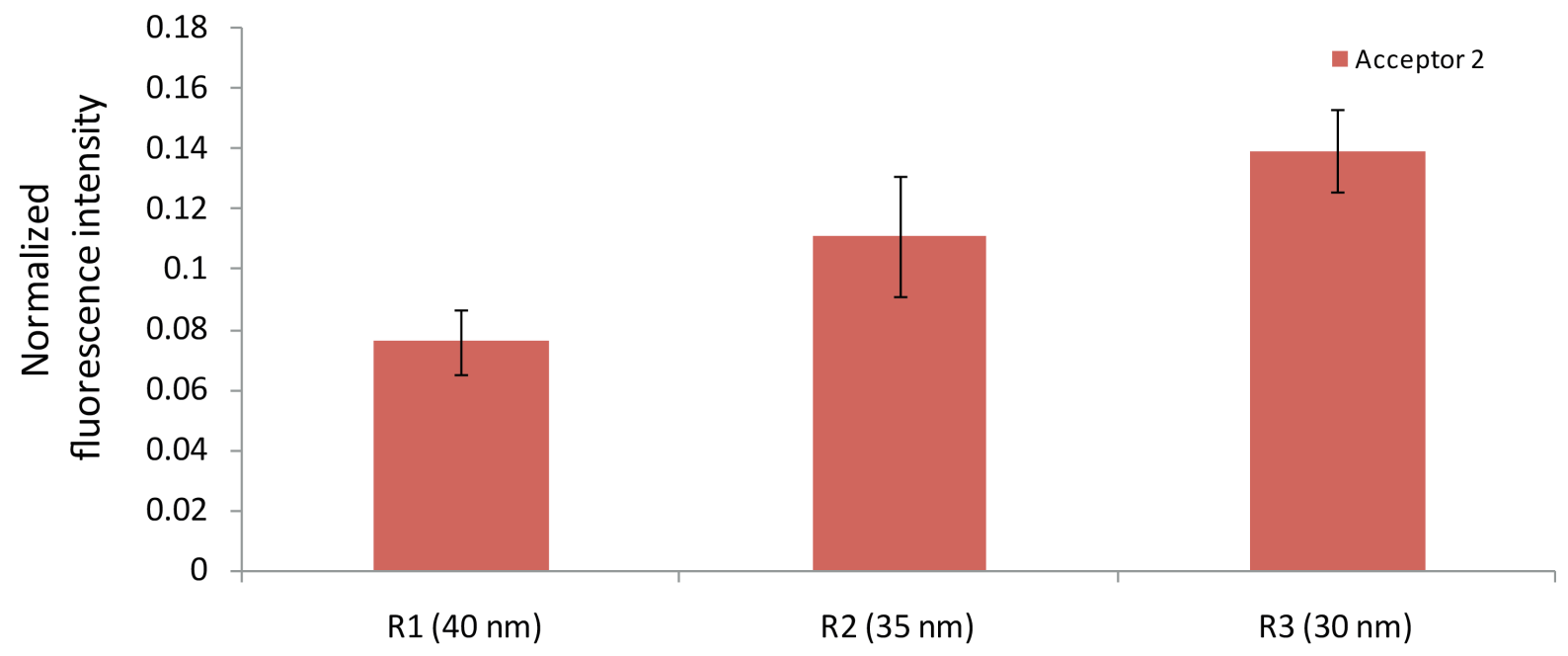

Figure S17 Study of position-dependent fluorescence intensity of acceptor 2. Polymer was aligned in Rturned conformation. 


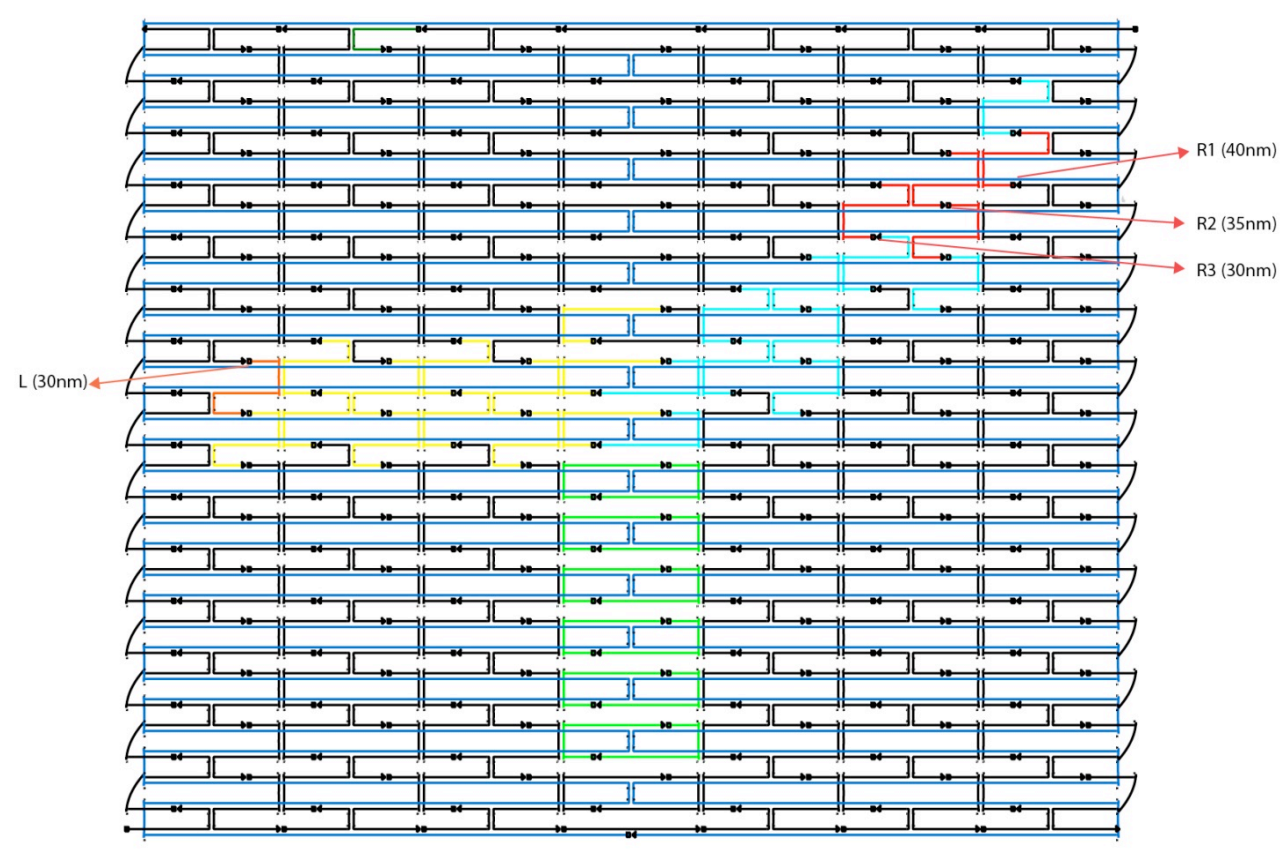

Figure S18 Illustration of DNA origami containing a 3-way intersection of ssDNA tracks made from short 5'-ssDNA-extended staple strands. Black represents non-extended staple strands. Middle, left and right tracks are represented in green, yellow and blue, respectively. Orange and red strands represent Alexa594 (acceptor 1) and Alexa647 (acceptor 2)-modified DNA, respectively. For distance-dependent fluorescence intensity experiment, Acceptor 2 (denoted R1-3) was placed at three different positions on DNA origami. The total distances of R1, R2 and R3 from middle track are 40, 35 and $30 \mathrm{~nm}$, respectively.

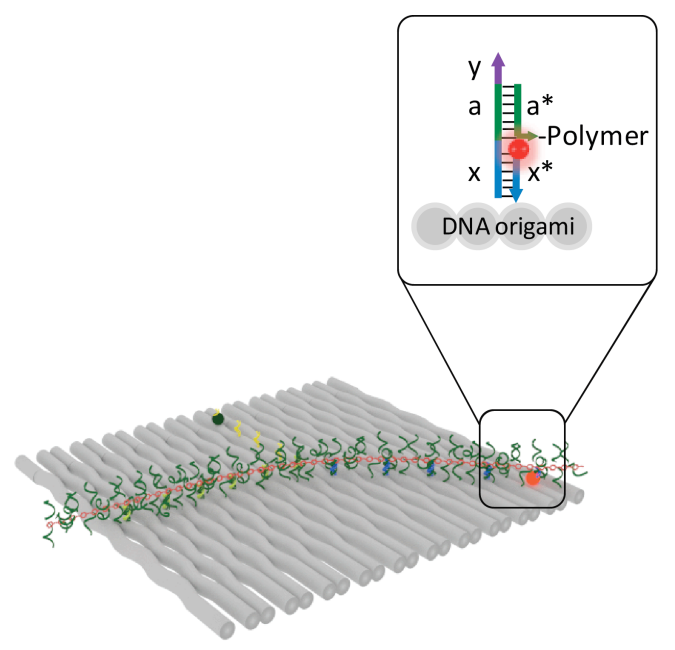

Figure S19 Illustration of the position of acceptor (red sphere)-modified staple strands and immobilized poly(APPV-DNA) in a R-turned pattern on DNA origami. 


\section{Supporting Information}

Table S2 Statistical analysis of controllable conformation of polymer aligned on DNA origami using guiding strands.

\begin{tabular}{|l|c|c|c|c|c|c|}
\hline \multirow{2}{*}{$\begin{array}{l}\text { DNA origami }+ \\
\text { poly(APPV-DNA) } \\
\text { linker strands }\end{array}$} & \multicolumn{4}{|c|}{ AFM images of poly(APPV-DNA) aligned on DNA origami } & \multirow{2}{*}{ Total } \\
\cline { 2 - 6 } & $\begin{array}{c}\text { Plain } \\
\text { origami }\end{array}$ & Straight line & $\begin{array}{c}\text { L-turned } \\
\text { pattern }\end{array}$ & $\begin{array}{c}\text { R-turned } \\
\text { pattern }\end{array}$ & $\begin{array}{c}\text { Misaligned } \\
\text { pattern }\end{array}$ & \\
\hline No linker strand & 32 & 94 & 4 & 0 & 19 & 149 \\
\hline LgDNA & 22 & 35 & 92 & 0 & 34 & 183 \\
\hline RgDNA & 10 & 17 & 4 & 75 & 39 & 145 \\
\hline
\end{tabular}

Table S3 Statistical analysis of polymer switching.

\begin{tabular}{|c|c|c|c|c|c|c|}
\hline \multirow{2}{*}{$\begin{array}{c}\mathrm{n}^{\text {th }} \text { of switching } \\
\text { (expected polymer pattern) }\end{array}$} & \multicolumn{5}{|c|}{ AFM images of poly(APPV-DNA) aligned on DNA origami } & \multirow[b]{2}{*}{ Total } \\
\hline & $\begin{array}{c}\text { Plain } \\
\text { origami }\end{array}$ & $\begin{array}{l}\text { Straight } \\
\text { line }\end{array}$ & $\begin{array}{l}\text { L-turned } \\
\text { pattern }\end{array}$ & $\begin{array}{c}\text { R-turned } \\
\text { pattern }\end{array}$ & $\begin{array}{l}\text { Misaligned } \\
\text { pattern }\end{array}$ & \\
\hline Initial state $(\mathrm{R})$ & 0 & 14 & 4 & 200 & 147 & 365 \\
\hline 1st switching (L) & 45 & 88 & 159 & 1 & 60 & 353 \\
\hline 2nd switching $(\mathrm{R})$ & 15 & 43 & 16 & 165 & 171 & 410 \\
\hline
\end{tabular}

We used counting statistic to determine the standard uncertainty for each event. Error bars in Fig. 3D and $4 \mathrm{~F}$ were obtained by error propagation based on these standard uncertainties. 
Supporting Information

Table S4 Sequences of non-extended staple strand for self-assembly of DNA origami

\begin{tabular}{|c|c|}
\hline Name & Sequence (5'---> 3') \\
\hline 1 & GCCACCACTCTTTTCATAATCAAATAGCAAGG \\
\hline 2 & TCATAGCTTGTAAAACGACGGCCAAAGCGCCA \\
\hline 3 & CTAATTTACCGTTTTTATTTTCATCTTGCGGG \\
\hline 4 & GGACGTTGAGAACTGGCTCATTATGCGCTAAT \\
\hline 5 & TGTAGCATAACTTTCAACAGTTTCTAATTGTA \\
\hline 6 & TCTTACCAACCCAGCTACAATTTTAAAGAAGT \\
\hline 7 & AAAACAAACTGAGAAGAGTCAATATACCTTTT \\
\hline 8 & ATACGTAAGAGGCAAAAGAATACACTGACCAA \\
\hline 9 & GGAATTACCATTGAATCCCCCTCACCATAAAT \\
\hline 10 & GTAAAAGACTGGTAATATCCAGAAATTCACCA \\
\hline 11 & AAAAAAGGCTTTTGCGGGATCGTCGGGTAGCA \\
\hline 12 & AGAGAATCAGCTGATAAATTAATGCTTTATTT \\
\hline 13 & TGGTTTTTCTTTCCAGTCGGGAAAAATCATGG \\
\hline 14 & CGACAACTTCATCATATTCCTGATCACGTAAA \\
\hline 15 & TGAGTTTCAAAGGAACAACTAAAGATCTCCAA \\
\hline 16 & AACCTACCGCGAATTATTCATTTCACATCAAG \\
\hline 17 & TAAGTCCTGCGCCCAATAGCAAGCAAGAACGC \\
\hline 18 & CCAGGCGCGAGGACAGATGAACGGGTAGAAAA \\
\hline 19 & CTAAAATAAGTATTAACACCGCCTCGAACTGA \\
\hline 20 & TTTAACGTTCGGGAGAAACAATAACAGTACAT \\
\hline 21 & GTTGTAGCCCTGAGTAGAAGAACTACATTCTG \\
\hline 22 & GATGGCAAAAGTATTAGACTTTACAAGGTTAT \\
\hline 23 & TAGAATCCCCTTTTTTAATGGAAACGGATTCG \\
\hline 24 & AGACAGTCTCATATGTACCCCGGTTTGTATAA \\
\hline 25 & GCGCAGAGATATCAAAATTATTTGTATCAGAT \\
\hline 26 & ACGCTCAACGACAAAAGGTAAAGTATCCCATC \\
\hline 27 & ACGAGTAGATCAGTTGAGATTTAGCGCCAAAA \\
\hline 28 & GAAGATCGTGCCGGAAACCAGGCAGTGCCAAG \\
\hline 29 & TTTCAACTACGGAACAACATTATTAACACTAT \\
\hline 30 & GAGCCGCCCCACCACCGGAACCGCTGCGCCGA \\
\hline 31 & GGCGATCGCGCATCGTAACCGTGCGAGTAACA \\
\hline 32 & GCTCATTTCGCGTCTGGCCTTCCTGGCCTCAG \\
\hline 33 & GTGAGCTAGCCCTTCACCGCCTGGGGTTTGCC \\
\hline 34 & GTTTGCCACCTCAGAGCCGCCACCGCCAGAAT \\
\hline 35 & AGAAGTGTCATTGCAACAGGAAAAAATCGTCT \\
\hline
\end{tabular}




\begin{tabular}{|c|c|}
\hline 36 & GCGAAACAAGAGGCTTTGAGGACTAGGGAGTT \\
\hline 37 & GTTTGAGGTCAGGCTGCGCAACTGTTCCCAGT \\
\hline 38 & CCAGACGACAAATTCTTACCAGTAGATAAATA \\
\hline 39 & TGAACAAAGATAACCCACAAGAATAAGACTCC \\
\hline 40 & CAAAATTAGGATAAAAATTTTTAGGATATTCA \\
\hline 41 & AATGCCCCATAAATCCTCATTAAAAGAACCAC \\
\hline 42 & ACCGTTCTGATGAACGGTAATCGTAATATTTT \\
\hline 43 & AGGTTTTGGCCAGTTACAAAATAAACAGGGAA \\
\hline 44 & TAGCCCTATTATTTACATTGGCAGCAATATTA \\
\hline 45 & GCTCCATGACGTAACAAAGCTGCTACACCAGA \\
\hline 46 & GAAATGGAAAACATCGCCATTAAACAGAGGTG \\
\hline 47 & CTTAAACAACAACCATCGCCCACGCGGGTAAA \\
\hline 48 & TCATTACCGAACAAGAAAAATAATAATTCTGT \\
\hline 49 & GGATTTAGTTCATCAATATAATCCAGGGTTAG \\
\hline 50 & GTTAAAATAACATTAAATGTGAGCATCTGCCA \\
\hline 51 & CAACGCAAAGCAATAAAGCCTCAGGATACATT \\
\hline 52 & CTCATCTTGGAAGTTTCCATTAAACATAACCG \\
\hline 53 & TAAATATTGAGGCATAGTAAGAGCACAGGTAG \\
\hline 54 & GCCACGCTTTGAAAGGAATTGAGGAAACAATT \\
\hline 55 & ATCAGAGAGTCAGAGGGTAATTGAACCAGTCA \\
\hline 56 & AGGCGGTCTCTTTAGGAGCACTAAACATTTGA \\
\hline 57 & TTCGCCATGGACGACGACAGTATCGTAGCCAG \\
\hline 58 & TTTCATGATGACCCCCAGCGATTAAGGCGCAG \\
\hline 59 & GCTCACAAGGGTAACGCCAGGGTTTTGGGAAG \\
\hline 60 & ACGGTCAATGACAAGAACCGGATATGGTTTAA \\
\hline 61 & GCGTTATACGACAATAAACAACATACAATAGA \\
\hline 62 & GCCAGCTGCGGTTTGCGTATTGGGAATCAAAA \\
\hline 63 & TTAAGACGATTAATTACATTTAACACAAAATC \\
\hline 64 & AGTAATCTTCATAAGGGAACCGAACTAAAACA \\
\hline 65 & CGTAACGAAAATGAATTTTCTGTAGTGAATTT \\
\hline 66 & TTGAATTATTGAAAACATAGCGATTATAACTA \\
\hline 67 & ATCAACAGGAGAGCCAGCAGCAAAATATTTTT \\
\hline 68 & TTATTAATGAACAAAGAAACCACCTTTTCAGG \\
\hline 69 & CTTTGAAAATAGGCTGGCTGACCTACCTTATG \\
\hline 70 & CCGGAAACTAAAGGTGAATTATCATAAAAGAA \\
\hline 71 & CTAAAGCAAATCAATATCTGGTCACCCGAACG \\
\hline 72 & AAAGATTCTAAATTGGGCTTGAGATTCATTAC \\
\hline 73 & ACAGAAATCTTTGAATACCAAGTTAATTTCAT \\
\hline 74 & TATCAGGGCGAAAATCCTGTTTGACGGGCAAC \\
\hline 75 & AAATCAATCGTCGCTATTAATTAAATCGCAAG \\
\hline 76 & AAAGGCCGCTCCAAAAGGAGCCTTAGCGGAGT \\
\hline
\end{tabular}




\begin{tabular}{|c|c|}
\hline 77 & TTATTCATGTCACCAATGAAACCATTATTAGC \\
\hline 78 & ATTATTTATTAGCGAACCTCCCGACGTAGGAA \\
\hline 79 & CCTGATTGAAAGAAATTGCGTAGAAGAAGGAG \\
\hline 80 & CATAACCCGCGTCCAATACTGCGGTATTATAG \\
\hline 81 & CAATGACAGCTTGATACCGATAGTCTCCCTCA \\
\hline 82 & TACATACACAGTATGTTAGCAAACTGTACAGA \\
\hline 83 & ACGCAAAGAAGAACTGGCATGATTTGAGTTAA \\
\hline 84 & AGTTTGGACGAGATAGGGTTGAGTGTAATAAC \\
\hline 85 & CCAAAATAAGGGGGTAATAGTAAAAAAAGATT \\
\hline 86 & GCCAACAGATACGTGGCACAGACATGAAAAAT \\
\hline 87 & AGGCGTTAGGCTTAGGTTGGGTTAAGCTTAGA \\
\hline 88 & TAAAGTACCAGTAGGGCTTAATTGCTAAATTT \\
\hline 89 & TTATTACGTAAAGGTGGCAACATACCGTCACC \\
\hline 90 & AATCACCACCATTTGGGAATTAGACCAACCTA \\
\hline 91 & CTTTCATCTCGCATTAAATTTTTGAGCAAACA \\
\hline 92 & TAACCTCCAATAAGAATAAACACCTATCATAT \\
\hline 93 & GCTAAATCCTTTTGCGGGAGAAGCCCGGAGAG \\
\hline 94 & TATATTTTCATACAGGCAAGGCAAAGCTATAT \\
\hline 95 & GAGGCGTTTCCCAATCCAAATAAGATAGCAGC \\
\hline 96 & AACCAGAGACCCTCAGAACCGCCACGTTCCAG \\
\hline 97 & ACAAAGAAAATTTCATCTTCTGACAGAATCGC \\
\hline 98 & CATATTTATTTCGAGCCAGTAATAAATCAATA \\
\hline 99 & GAATGGCTACCAGTAATAAAAGGGCAAACTAT \\
\hline 100 & AGTGTACTATACATGGCTTTTGATCTTTCCAG \\
\hline 101 & ACTGCCCGCTTTTCACCAGTGAGATGGTGGTT \\
\hline 102 & ATTAAGTTTTCCACACAACATACGCCTAATGA \\
\hline 103 & TGGACTCCGGCAAAATCCCTTATACGCCAGGG \\
\hline 104 & ACTGGATATCGTTTACCAGACGACTTAATAAA \\
\hline 105 & GCAAATATGATTCTCCGTGGGAACCGTTGGTG \\
\hline 106 & CTTGCATGCCGAGCTCGAATTCGTCCTGTCGT \\
\hline 107 & GGTAGCTATTGCCTGAGAGTCTGGTTAAATCA \\
\hline 108 & CATGTCAAAAATCACCATCAATATAACCCTCA \\
\hline 109 & CTTTACAGTATCTTACCGAAGCCCAGTTACCA \\
\hline 110 & CCAAATCATTACTTAGCCGGAACGTACCAAGC \\
\hline 111 & ACCCGTCGTTAAATTGTAAACGTTAAAACTAG \\
\hline 112 & AAACGAAATGCCACTACGAAGGCAGCCAGCAA \\
\hline 113 & TCGGTTTAGGTCGCTGAGGCTTGCAAAGACTT \\
\hline 114 & CGATTTTAGGAAGAAAAATCTACGGATAAAAA \\
\hline 115 & GTCACACGATTAGTCTTTAATGCGGCAACAGT \\
\hline 116 & ACGGCTACAAGTACAACGGAGATTCGCGACCT \\
\hline 117 & ATATATTCTCAGCTTGCTTTCGAGTGGGATTT \\
\hline
\end{tabular}




\begin{tabular}{|c|c|}
\hline 118 & GACTTGAGGTAGCACCATTACCATATCACCGG \\
\hline 119 & AATGGTTTTGCTGATGCAAATCCATTTTCCCT \\
\hline 120 & ACGAACTATTAATCATTGTGAATTTCATCAAG \\
\hline 121 & CACGACGTGTTTCCTGTGTGAAATTTGCGCTC \\
\hline 122 & CGGAATTACGTATTAAATCCTTTGGTTGGCAA \\
\hline 123 & AGCTGATTACTCACATTAATTGCGTGTTATCC \\
\hline 124 & TATGTAAAGAAATACCGACCGTGTTAAAGCCA \\
\hline 125 & CACCAGAGTTCGGTCATAGCCCCCTCGATAGC \\
\hline 126 & CAATAAATAAATGCAATGCCTGAGAAGGCCGG \\
\hline 127 & TAGATGGGGTGCGGGCCTCTTCGCGCAAGGCG \\
\hline 128 & TGCCTTGACAGTCTCTGAATTTACCCCTCAGA \\
\hline 129 & GAGAATAGGTCACCAGTACAAACTCCGCCACCCTCAGAGC \\
\hline 130 & TACTCAGGAGGTTTAGATAGTTAG \\
\hline 131 & GAAGGATTAGGATTAGAAACAGTT \\
\hline 132 & TGCTAAACTCCACAGACAGCCCTCTACCGCCACCCTCAGA \\
\hline 133 & TAAGCGTCGGTAATAAGTTTTAACCCGTCGAGAGGGTTGA \\
\hline 134 & ACAAACAACTGCCTATTTCGGAACCTGAGACTCCTCAAGA \\
\hline 135 & CACCACCCTCATTTTCCGTAACAC \\
\hline 136 & GGAAAGCGGTAACAGTGCCCGTATCGGGGTTTTGCTCAGT \\
\hline 137 & ACCAGGCGGATAAGTGGGGGTCAG \\
\hline 138 & ACCGCCACCCTCAGAAACAACGCC \\
\hline 139 & TATAAGTATAGCCCGGAATAGGTGTATCACCG \\
\hline 140 & ACGTTAGTTCTAAAGTTTTGTCGTGATACAGG \\
\hline 141 & GCGTACTATGGTTGCTAATTAACC \\
\hline 142 & ACGTGCTTTCCTCGTTGCCACCGA \\
\hline 143 & CTAAACAGGAGGCCGAGAATCCTG \\
\hline 144 & GAATAGCCACAAGAGTCCACTATTAAGCCGGCGAACGTGG \\
\hline 145 & CTAAATCGGAACCCTAAAACCGTC \\
\hline 146 & CCAGCAGGCGATGGCCCACTACGTGAGGTGCCGTAAAGCA \\
\hline 147 & CGAGAAAGGAAGGGAATGCGCCGCTACAGGGC \\
\hline 148 & CCGCCAGCTTTTATAATCAGTGAGAGAATCAGAGCGGGAG \\
\hline 149 & CCGAAATCAACGTCAAAGGGCGAAAAGGGAGCCCCCGATT \\
\hline 150 & CGGCCTTGGTCTGTCCATCACGCATTGACGAGCACGTATA \\
\hline 151 & TAGAGCTTGACGGGGAAAAGAACG \\
\hline 152 & ATCACTTGAATACTTCTTTGATTAGTTGTTCC \\
\hline 153 & GAATAAGGTTTTAAATTGTGTCGAAATCTGTATCAT \\
\hline 154 & AAACAGTTTTTTTAATGCAGATACATAAGAATACCA \\
\hline 155 & GCATAAAGTTTTGAAAGGGGGATGTGCTTATTACGC \\
\hline 156 & CGCCTGATTTTTGACAGCATCGGAACGAACCCTCAG \\
\hline 157 & GATTGACCTTTTCCCAAAAACAGGAAGATGATAATC \\
\hline 158 & CATTCAACTTTTCTTGCCCTGACGAGAACATTCAGT \\
\hline
\end{tabular}


Supporting Information

\begin{tabular}{|l|l|}
\hline 159 & CAGCGAAATTTTTTTTTTCACGTTGAAAGAATTGCG \\
\hline 160 & AGGTAAAGTTTTACTAATAGTAGTAGCAAGGTGGCA \\
\hline 161 & TTTTCAAGTTTTTGGGGTCGAACCATC \\
\hline 162 & GAGTTGCATTTTTGTAAAGCCTGGGGTGAGCCGGAA \\
\hline 163 & TCAATTCTTTTTGATGGCTTAGAGCTTAAGAGGTCA \\
\hline 164 & AATAATAATTTTATAGGAACCCATGTACAGGGATAGCAAGCCCA \\
\hline 165 & AGAAAAGCTTTTATTCAAAAGGGTGAGATAATGTGT \\
\hline 166 & CAGCTGGCTTTTGTAATGGGATAGGTCAAAACGGCG \\
\hline 167 & TTTTTGCGTTTTCAGAAAACGAGAATGAAATGCTTT \\
\hline 168 & ACCCAAATTTTTGCAAGCGGTCCACGCTCCCTGAGA \\
\hline 169 & CTGAAACATTTTGTCAGACGATTGGCCTCAGGAGGT \\
\hline 170 & TGCCTTTATTTTAGACAAAAGGGCGACAGGTTTACC \\
\hline 171 & TGAGGCAGTTTTGCGTCAGACTGTAGCGATCAAGTT \\
\hline 172 & GAAGGAAAAATAGAAAATTCATATTTCAACCG \\
\hline 173 & TTTTGTTTTTTTCTTATCCGGTATTCTAAATCAGA \\
\hline 174 & AAAAGTAATTTTAACGTCAAAAATGAAAAAACGATT \\
\hline 175 & TTTTTGAAAGTATTAAGAGGCTATTATT \\
\hline 176 & GGAAATACTTTTCAGGAACGGTACGCCATTAAAGGGATTTTAGA \\
\hline 177 & TATAGAAGTTTTACGCGCCTGTTTATCAGTTCAGCT \\
\hline 178 & TGGATTATTTTTGCCGTCAATAGATAATCAACTAAT \\
\hline 179 & ACGAACCATTTTCTACATTTTGACGCTCACGCTCAT \\
\hline 180 & AGCGCCAATTTTGCAGATAGCCGAACAATTTTTAAG \\
\hline 181 & AGATTAGATTTTCCAGCAGAAGATAAAAAATACCGA \\
\hline 182 & ATCAAAATTTTTGAAGATGATGAAACAAAATTACCT \\
\hline 183 & AATGCAGATTTTGAAAAAGCCTGTTTAGGGAATCAT \\
\hline 184 & AATTACTATTTTCATAGGTCTGAGAGACGTGAATTT \\
\hline 185 & GAGCAAAATTTTACTTCTGAATAATGGATGATTGTT \\
\hline & \\
\hline
\end{tabular}


Table S5 Sequences of ssDNA-extended and fluorophore-tagged staple strands for self-assembly of DNA origami structures containing ssDNA track. Black letters represent the part of the staple strand hybridized to the scaffold strand. Green letters represent staple strand extensions that are complementary to the polymer handle. Yellow and blue are partially complementary to LgDNA and RgDNA, respectively.

\begin{tabular}{|c|c|}
\hline Name & Sequence (5'---> 3') \\
\hline 3wayM_1 & GTTAGATGATTTGGGAGAGGCATTAATGAATCGGCCACCTGAAA \\
\hline 3wayM_2 & GTTAGATGATTTCCCGGGTACCTGCAGGTCGACTCTCAAATATC \\
\hline 3wayM_3 & GTTAGATGATTTCTGTAAATATATGTGAGTGAATAAAAAGGCTA \\
\hline 3wayM_4 & GTTAGATGATTTCTTTTACACAGATGAATATACAGTGCCATCAA \\
\hline 3wayM_5 & GTTAGATGATTTGCGTAAGAAGATAGAACCCTTCTGAACGCGCG \\
\hline 3wayM_6 & GTTAGATGATTTCTGTAATAGGTTGTACCAAAAACACAAATATA \\
\hline 3wayM_7 & GTTAGATGATTTGCTTCTGGCACTCCAGCCAGCTTTACATTATC \\
\hline 3wayM_8 & GTTAGATGATTTTCAGGTCATTTTTGAGAGATCTACCCTTGCTT \\
\hline 3wayM_9 & GTTAGATGATTTTTTTAGTTCGCGAGAAAACTTTTTTTATGACC \\
\hline 3wayM_10 & GTTAGATGATTTAAATAATTTTTAACCAATAGGAACAACAGTAC \\
\hline 3wayM_11 & GTTAGATGATTTATTTTGCGTTTAAAAGTTTGAGTACCGGCACC \\
\hline 3wayM_12 & GTTAGATGATTTAAACCCTCTCACCTTGCTGAACCTAGAGGATC \\
\hline 3wayL_1 & ACTACTCAA TTTCAAAAATCATTGCTCCTTTTGATAATTGCTGA \\
\hline 3wayL_2 & ACTACTCAATTTATATAATGGGGGCGCGAGCTGAAATTAACATC \\
\hline 3wayL_3 & ACTACTCAATTTAAGAGGAACGAGCTTCAAAGCGAAAGTTTCAT \\
\hline 3wayL_4 & ACTACTCAATTTTTTCATTTCTGTAGCTCAACATGTTTAGAGAG \\
\hline 3wayL_5 & ACTACTCAATTTGAAGCAAAAAAGCGGATTGCATCAATGTTTAG \\
\hline 3wayL_6 & ACTACTCAA TTTTTTGCCAGGCGAGAGGCTTTTGCAATCCTGAA \\
\hline 3wayL_7 & ACTACTCAATTTTACCTTTAAGGTCTTTACCCTGACAATCGTCA \\
\hline 3wayL_8 & ACTACTCAATTTTCGCAAATAAGTACGGTGTCTGGACCAGACCG \\
\hline 3wayL_9 & ACTACTCAATTTTCAGAAGCCTCCAACAGGTCAGGATTTAAATA \\
\hline 3wayL_10 & ACTACTCAATTTTCCATATATTTAGTTTGACCATTAAGCATAAA \\
\hline 3wayL_11 & ACTACTCAA TTTTGCAACTAGGTCAATAACCTGTTTAGAATTAG \\
\hline 3wayL_12 & ACTACTCAATTTTTTTAATTGCCCGAAAGACTTCAACAAGAACG \\
\hline 3wayL_13 & ACTACTCAATTTCGAGTAGAACAGTTGATTCCCAATATTTAGGC \\
\hline 3wayR_1 & СТCСACTCCTTTATTGAGGGAATCAGTAGCGACAGACGTTTTCA \\
\hline 3wayR_2 & СТССАСТССТTTTCACAATCCCGAGGAAACGCAATAATGAAATA \\
\hline 3wayR_3 & СТССАСТССТTTATACCCAAACACCACGGAATAAGTGACGGAAA \\
\hline 3wayR_4 & СTCCACTCCTTTGCCCAATAGACGGGAGAATTAACTTTCCAGAG \\
\hline 3wayR_5 & СTCCACTCCTTTCAAGCAAGCGAGCATGTAGAAACCAGAGAATA \\
\hline 3wayR_6 & СТCСACTCСTTTGCGCATTAATAAGAGCAAGAAACAATAACGGA \\
\hline 3wayR_7 & СТCСACTCCTTTAGCACCGTAGGGAAGGTAAATATTTTATTTTG \\
\hline 3wayR_8 & CTCCACTCCTTTTATTTTGCACGCTAACGAGCGTCTGAACACCC \\
\hline 3wayR_9 & СТССАСТССТTTGCAATAGCAGAGAATAACATAAAAACAGCCAT \\
\hline
\end{tabular}




\begin{tabular}{|l|l|}
\hline 3wayR_10 & CTCCACTCCTTTAGAGGCATACAACGCCAACATGTATCTGCGAA \\
\hline 3wayR_11 & CTCCACTCCTTTATCGGCTGACCAAGTACCGCACTCTTAGTTGC \\
\hline 3wayR_12 & CTCCACTCCTTTCCTAATTTAAGCCTTAAATCAAGAATCGAGAA \\
\hline 3wayR_13 & CTCCACTCCTTTGGTATTAATCTTTCCTTATCATTCATATCGCG \\
\hline 3wayR_14 & CTCCACTCCTTTTCGGCATTCCGCCGCCAGCATTGATGATATTC \\
\hline 3wayL_1_Alexa594 & [Alexa594] CTACTCAATTTCAAAAATCATTGCTCCTTTTGATAATTGCTGA \\
\hline 3wayR_1_Alexa647 & [Alexa647]CTCCACTCCTTTATTGAGGGAATCAGTAGCGACAGACGTTTTCA \\
\hline 3wayR_2_Alexa647 & [Alexa647]CTCCACTCCTTTTCACAATCCCGAGGAAACGCAATAATGAAATA \\
\hline 3wayR_3_Alexa647 & [Alexa647]CTCCACTCCTTTATACCCAAACACCACGGAATAAGTGACGGAAA \\
\hline
\end{tabular}

Table S6 Sequences of guiding and removing strands

\begin{tabular}{|l|l|}
\hline Name & Sequence $\left(5^{\prime}--->3^{\prime}\right)$ \\
\hline LgDNA & TTGAGTAGTGTTAGATGAGTTAGG \\
\hline LrDNA & CCTAACTCATCTAACACTACTCAA \\
\hline RgDNA & GGAGTGGAGGTTAGATGAGGTGAG \\
\hline RrDNA & CTCACCTCATCTAACCTCCACTCC \\
\hline
\end{tabular}

\section{References}

1. Knudsen, J. B.; Liu, L.; Bank Kodal, A. L.; Madsen, M.; Li, Q.; Song, J.; Woehrstein, J. B.; Wickham, S. F. J.; Strauss, M. T.; Schueder, F. et al. Routing of Individual Polymers in Designed Patterns. Nat. Nanotechnol. 2015, 10, 892-898.

2. Preus, S., a|e - UV-Vis-IR Spectral Software 1.2, FluorTools, www.fluortools.com 